\title{
AN INNOVATIVE ROUTE FROM WIND TUNNEL EXPERIMENTS TO FLIGHT DYNAMICS ANALYSIS FOR A HIGHLY SWEPT FLYING WING
}

\author{
J. Schwithal ${ }^{1}$, D. Rohlf \\ DLR Institute of Flight Systems, \\ Lilienthalplatz 7, 38108 Braunschweig, Germany \\ G. Looye \\ DLR Institute of System Dynamics and Control, \\ Münchener Straße 20, 82234 Weßling, Germany \\ C. M. Liersch \\ DLR Institute of Aerodynamics and Flow Technology, \\ Lilienthalplatz 7, 38108 Braunschweig, Germany
}

\begin{abstract}
A flying wing configuration with highly swept leading edges and low aspect ratio such as the generic UCAV configuration DLR-F19 is very attractive for military applications due to its very favorable stealth capabilities as well as its high agility. To assure good flying qualities, however, is a critical aspect for such a configuration. It should thus be considered early in the design process. This paper presents an innovative way to derive a flight dynamics model from wind tunnel experiments by applying a system identification approach, normally employed for flight tests. This allows the modelling of nonlinear aerodynamic effects and provides a model which can be integrated directly into flight dynamics simulations. New wind tunnel maneuvers are applied, which significantly reduce the time of the wind tunnel experiments and improve the quality of the aerodynamic dataset generation. The aerodynamic model is then integrated into a 6-degreesof-freedom simulation environment in order to perform a flight dynamics analysis of the UCAV configuration. The purpose of this analysis is to compare the flying qualities as derived from wind tunnel data with the numerical results determined on the basis of potential flow methods used in preliminary aircraft design.
\end{abstract}

\section{Keywords}

system identification, parameter estimation, flying qualities analysis, wind tunnel experiment, aerodynamic modelling, UCAV

\section{Acknowledgments}

The authors would like to thank all colleagues of the DLR project FaUSST who contributed to the work presented here. They especially appreciate the contributions of the colleagues of the DNW-NWB for performing the wind tunnel experiments and the colleagues from the DLR Institute of Propulsion Technology for providing the engine performance map for the UCAV model.

\footnotetext{
${ }^{1}$ Corresponding author: e-mail: jana.schwithal@dlr.de, telephone: +49 531 295-3255, fax: +49 531 295-2845
} 


\section{LIST OF SYMBOLS}

$\alpha \quad$ angle of attack, $\left[{ }^{\circ}\right]$

$a^{*} \quad$ angle of attack where half of airflow is

detached, $\left[^{\circ}\right]$

$\beta \quad$ angle of sideslip, [ $\left.{ }^{\circ}\right]$

$\Delta \alpha \quad$ angle of attack difference between current and next breakpoint, $\left[{ }^{\circ}\right]$

$\Delta C_{\mathrm{i}}^{\mathrm{j}} \quad$ delta coefficient of the force or moment $\mathrm{i}$ whose strongest influence is parameter $\mathrm{j},[-]$ equivalent time delay of parameter $\mathrm{i},[\mathrm{s}]$

$\Delta t_{i}$

$\Phi \quad$ bank angle, $\left[^{\circ}\right]$

$\Phi_{t} \quad$ critical bank angle for roll performance, $\left[^{\circ}\right]$

$\omega_{0} \quad$ natural frequency, $[\mathrm{rad} / \mathrm{s}]$

$a_{1}$

$B p$

$C_{\mathrm{i}}$

$C_{\mathrm{i} 0}$

$C_{\mathrm{ij}}$

$C_{i j \alpha}$

$C_{D X}, C_{m X}$ hysteresis influence factor on drag and

$D \quad$ pitching moment,

$F_{V D} \quad$ vortex drag factor, [-]

$f_{0} \quad$ model oscillation frequency, $[\mathrm{Hz}]$

$f_{s} \quad$ sampling frequency, $[\mathrm{Hz}]$

g gravity constant, $\left[\mathrm{m} / \mathrm{s}^{2}\right]$

$I_{x x}, I_{y y}, I_{z z} \quad$ moment of inertia in $x / y / z$-axis, $\left[\mathrm{kg} \mathrm{m}^{2}\right]$

$L, D, Y \quad$ aerodynamic lift, drag and side force [N]

$L_{\beta}, N_{\beta}$

$L I B$

$\angle O B$

$I, m, n$

$N_{r}$

$n_{z}$

$p, q, r$

$V$

$R I B$

$R O B$

SP20

SP25

$T_{2}$

$t$

$x$

$x, y, z$

$Y_{\beta}, Y_{\text {rud }}$ dimensional roll moment derivative with respect to sideslip angle, $\left[1 / \mathrm{s}^{2}\right]$

left inboard control surface, $\left[^{\circ}\right]$

left outboard control surface, $\left[^{\circ}\right]$

aerodynamic moments, [N m]

dimensional yaw moment derivative with respect to roll rate, $[1 / \mathrm{s}]$

vertical load factor, [-]

roll rate, pitch rate, yaw rate, $[\mathrm{rad} / \mathrm{s}]$

velocity $[\mathrm{m} / \mathrm{s}]$

right inboard control surface, $\left[{ }^{\circ}\right]$

right outboard control surface, $\left[^{\circ}\right]$

split flap with $20 \%$ chord depth, $\left[{ }^{\circ}\right]$

split flap with $25 \%$ chord depth, $\left[^{\circ}\right]$

time to double amplitude, [s]

time, [s]

non-dimensional flow separation point on chord, [-]

longitudinal, lateral, vertical position [m]

dimensional side force derivative with respect to sideslip angle and rudder deflection, [1/s]

\section{LIST OF ABBREVIATIONS}

$\begin{array}{ll}\text { AC } & \text { AirCraft } \\ \text { AVT } & \text { Applied Vehicle Technology } \\ \text { CAP } & \text { Control Anticipation Parameter } \\ \text { CFD } & \text { Computational Fluid Dynamics } \\ \text { CPACS } & \text { Common Parametric Aircraft Configuration } \\ & \text { Schema } \\ \text { DAMIP } & \text { Dynamic Aircraft Model Integration Process } \\ \text { DNW } & \text { German-Dutch Wind Tunnels } \\ \text { DoF } & \text { Degrees of Freedom } \\ \text { HAREM } & \text { HAndling qualities Reasearch using Matlab }\end{array}$

LSP Left SPlit flap

MPM Model Positioning Mechanism

NASA National Aeronautics and Space

Administration

NATO North Atlantic Treaty Organization

NWB Low-Speed Wind Tunnel Braunschweig

RANS Reynolds-averaged Navier-Stokes

RSP Right SPlit flap

RTO Research and Technology Organisation

SACCON Stability And Control CONfiguration

STO Science and Technology Organization

SysID System IDentification

UCAV Unmanned Combat Aerial Vehicle

\section{LIST OF INDICES}

$\begin{array}{ll}\text { a } & \text { aerodynamic coordinate system } \\ \text { ail } & \text { aileron } \\ \text { b } & \text { body-fixed coordinate system } \\ \text { dr } & \text { Dutch roll } \\ \text { hys } & \text { hysteresis } \\ \text { rud } & \text { rudder } \\ \text { SP } & \text { short period }\end{array}$

\section{INTRODUCTION}

The DLR-F19 configuration (Fig. 1) is a highly swept flying wing with a low aspect ratio and a partially round and partially sharp leading edge. It has the same lambda-wing planform as the SACCON (Stability And Control CONfiguration) wind tunnel model built by NASA, but different control surfaces. The configuration was established within the NATO/RTO Task group AVT-161 "Assessment of Stability and Control Prediction Methods for NATO Air and Sea Vehicles" [1] and has extensively been analyzed concerning different disciplines at DLR.

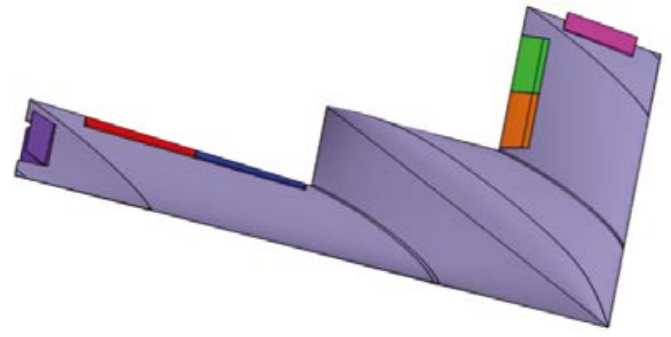

Fig. 1 DLR-F19 configuration

A detailed description of the configuration and its design process can be found in [2]. A large benefit of the configuration is its favorable stealth capability resulting from its planform with parallel edges, which makes it an attractive candidate as a future UCAV (Unmanned Combat Aerial Vehicle) for military applications. Concerning the aerodynamic analysis and the assurance of an adequate flight dynamic behavior, however, the configuration is quite challenging. The flow field around the configuration is dominated by vortex structures and vortex-to-vortex interactions. The aerodynamic behavior is thus strongly nonlinear and difficult to predict with analytical approaches. Wind tunnel experiments have been conducted to study these complex aerodynamics and to provide a solid aerodynamic database.

In order to use these data of the wind tunnel experiments for flight dynamics simulations, they have to be converted into an aerodynamic model first. In the case of nonlinear 
aerodynamics this is not trivial. The approach presented in this paper is based on a system identification method, as it is usually employed for flight tests, to determine the static and dynamic derivatives of the configuration and to develop an aerodynamic model.

Static or stability derivatives are the rates of change of aerodynamic force and moment coefficients with respect to linear or angular velocity components. Dynamic derivatives are the time derivatives thereof. They are needed for the determination of stability and contro characteristics of an aircraft. They are also required for load assessments of individual airplane components and finally for the validation of numerical codes.

Dynamic derivatives are usually tested in wind tunnels incorporating special wind tunnel models as well as dedicated test rigs enabling sinusoidal oscillations of the model at certain reference conditions. A classical method for the modelling of the dynamic derivatives is to employ linear aerodynamic models. However, this approach is not ideally suited for an extended flight envelope as already outlined in [3]. Furthermore, it is rather time consuming especially when testing numerous model configurations e.g. to determine the effectiveness of a variety of control surfaces. Therefore the conventional sinusoidal oscillations in the wind tunnel experiments have been replaced with newly designed large amplitude maneuvers including superimposed frequency sweeps. Compared to [3], the applied system identification model has been significantly modified and enhanced in order to determine static and dynamic derivatives as well as the effectiveness of control surfaces. The distinguished nonlinear aerodynamic model is supposed to be directly applicable for succeeding simulation purposes.

An alternative approach to model the aerodynamic behavior of an aircraft is the numerical calculation of the aerodynamic coefficients. When using RANS (ReynoldsAveraged Navier-Stokes) based high-fidelity CFD methods, these computations can be very expensive in terms of time and computational costs. Another alternative, typically used in the early phases of aircraft design, is to use methods which are based on simplified flow equations. One of these methods is VSAERO [4], a classical 3D panel method based on the linearized potential flow equation. By using such a fast and robust method, it is possible to create a comprehensive database for flight dynamics investigations automatically and within only a few hours of time. Certainly the aerodynamic dataset generated with VSAERO is not able to cover all relevant effects of the complex aerodynamics of the considered UCAV configuration. Nevertheless, it shall be applied here in order to investigate whether this approach is suitable to get first insights of the flight dynamic behavior of the aircraft - at least for low angles of attack, before the flow field is dominated by complex vortex structures. If so, this would be very beneficial for the early stages of design, when the geometry still changes permanently.

A comparison between the aerodynamic data determined with VSAERO and the aerodynamic dataset derived from the wind tunnel experiments is accomplished by applying different flying qualities criteria to flight dynamics models created from the two different datasets.

For the numerical analysis of the UCAV configuration the aircraft is modelled in the CPACS (Common Parametric
Aircraft Configuration Schema) data format [5]. CPACS is a parametric, hierarchical structure for the description of aircraft related data, which was developed at DLR. It serves as an interface and a common language allowing the exchange of information between different tools within the aircraft design process. The VSAERO computations as well as the generation of the flight dynamics model and the flying qualities analysis presented in sections 4.2 and 4.3 are all performed on the basis of the common CPACS file containing the UCAV configuration.

\section{EXPERIMENTAL SET-UP}

\subsection{Wind Tunnel Test Facility}

The tests described herein have been performed in the Low-Speed Wind Tunnel Braunschweig (NWB) of the German-Dutch Wind Tunnels (DNW). The DNW-NWB is an atmospheric low-speed wind tunnel, which has recently been refurbished to become an aero-acoustic facility. Detailed information about the DNW-NWB can be found in [6], [7] and [8].

The model is mounted by means of a ventral sting on NWB's Model Positioning Mechanism MPM (Fig. 2). Forces and moments are measured by a six component balance mounted internally in the model, whereas the position and orientation of the model are determined by a camera optical position measurement. The MPM can be described as a 6-degrees-of-freedom (DoF) parallel kinematics system incorporating six struts of constant length. The struts connect a Stewart platform to six electric linear motors at the wind-tunnel-fixed side providing three translatory and three rotatory degrees of freedom. The electric linear motors traverse along two rails, which are, like the Stewart platform, located above the test section. The MPM can be employed in combination with the open or closed test section configuration. The MPM can be used for high precision static model positioning as well as for arbitrary pre-defined maneuvers within its working space including e.g. sinusoidal model oscillations.

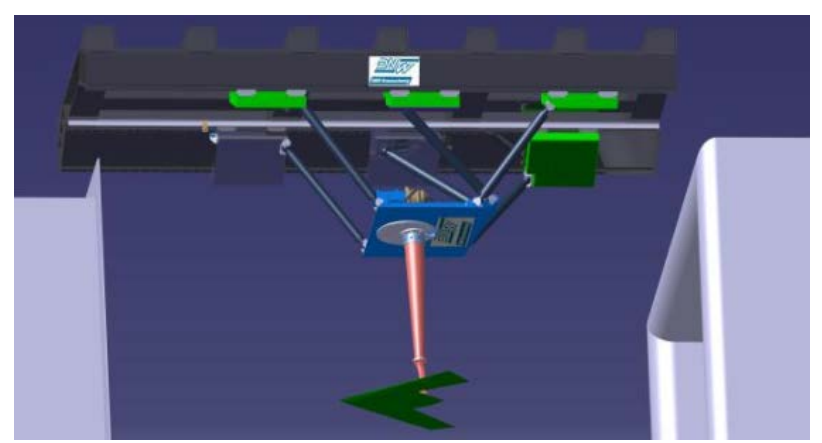

Fig. 2 Sketch of the Model Positioning Mechanism (MPM) in the DNW-NWB wind tunnel

In order to augment the working space about the modelfixed pitch and roll axes an additional electric actuator is mounted on the Stewart platform. The actuator drives a pushrod which is connected to dedicated balance mounts inside the model to convert the pushrod motion into the desired model oscillation. This so called " $7^{\text {th }}$ axis" is controlled by the same software as the linear motors. The frequency can be set continuously from $f_{0}=0.0 \mathrm{~Hz}$ to $f_{0}=3.0 \mathrm{~Hz}$. In general, the amplitude range depends on the frequency, the oscillation type and on the balance mount. The development of the dynamic testing systems 
at DNW-NWB, leading finally to the MPM, is described in [9].

\subsection{Flying Wing Model}

\subsubsection{Wind Tunnel Model}

The DLR-F19 wind tunnel model is a generic UCAV configuration with a $53^{\circ}$ leading edge sweep and a lambda wing planform, as shown in Fig. 3 . The model has been made from carbon fiber composite material in order to keep the model weight as low as possible, consequently reducing inertial forces and moments. The combination of high stiffness and low weight also leads to flexible eigenmode frequencies which are in the order of one magnitude above the intended rigid-body oscillation frequencies.

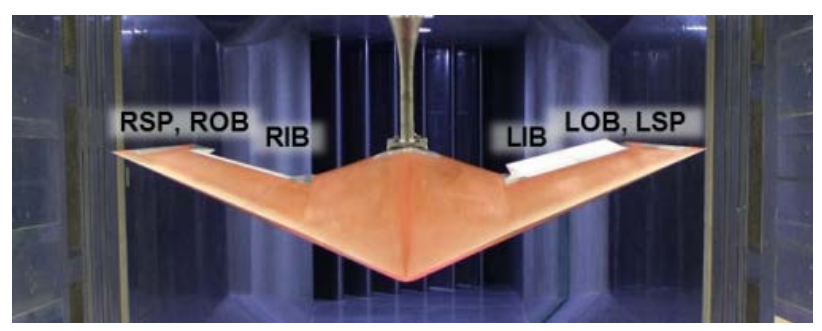

Fig. 3 DLR-F19 in the DNW-NWB closed test section (mounted top down on a belly sting). LIB/LOB label the left inboard/outboard control surfaces and RIB/ROB the right inboard/outboard control surfaces, respectively. RSP/LSP stands for right and left split flaps.

The model has a wing span of $1.54 \mathrm{~m}$ and a wing area of $0.77 \mathrm{~m}^{2}$. This corresponds to a scaling of 1:10 compared to full-size model of the aircraft. The wind tunnel model possesses two trailing edges at each wing, which cover $25 \%$ of the wing chord. At the sides of each wing is a pair of split flaps (Fig. 1 and 3). The split flaps can be exchanged for the wind tunnel experiments. One set of split flaps has a depth of $20 \%$ of the chord depth, the second pair a depth of 25\%. A 3D view of the model without split flaps is shown in Fig. 4.

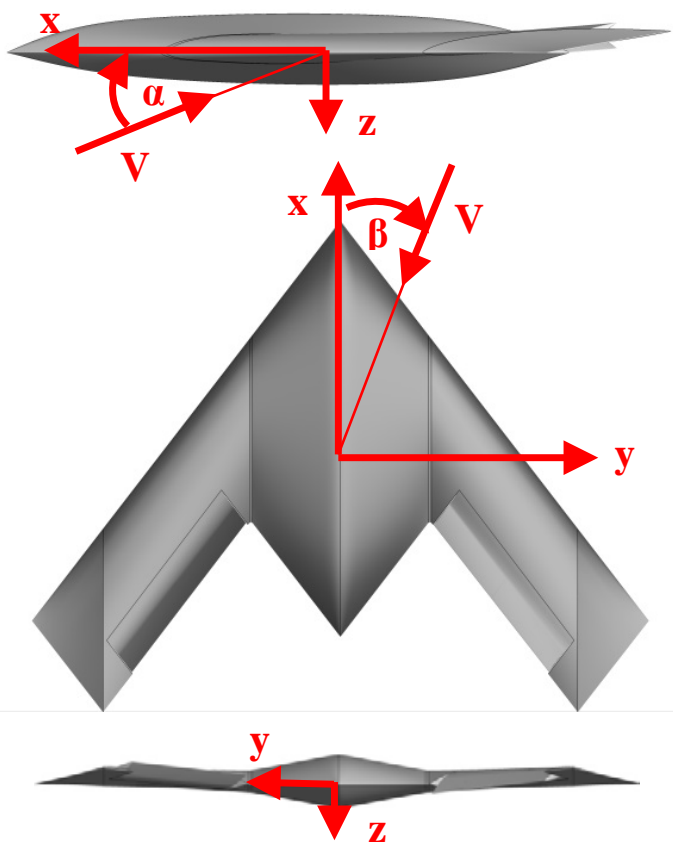

Fig. 4 Three-side-view of DLR-19 wind tunnel model
The design and manufacture of the DLR-F19 model as well as the wind tunnel tests at DNW-NWB have been performed within the NATO STO AVT-201 Task Group "Extended Assessment of Stability and Control Prediction Methods for NATO Air Vehicles" [10], [11].

\subsubsection{Model for Flying Qualities Analysis}

The considered aircraft configuration for the flying qualities analysis corresponds to the DLR-F19 UCAV configuration described above. The underlying wind tunnel data, however, result from the predecessor wind tunnel model, the NASA built SACCON. This model has an identical outer shape but smaller control surfaces on the trailing edge compared to the DLR-F19 configuration. The control surfaces of the SACCON model only cover $20 \%$ of the wing chord compared to $25 \%$ for the DLR-F19 model. The CPACS model of the DLR-F19 configuration for the VSAERO computations was thus also defined with control surfaces with $20 \%$ chord depth in order to have the same control surfaces size as the SACCON model. The effect of these slightly smaller control surfaces is a reduced control surface efficiency that has to be kept in mind when considering the results of section 4.3. Split flaps are not used in the flying qualities analysis.

The flying qualities analysis of the UCAV configuration is performed for the full-size model of the aircraft with the characteristics shown in Table 1. Actuator dynamics as well as automatic flight control are not considered for the flying qualities analysis.

\begin{tabular}{lc}
\hline Aircraft Parameter & Value \\
\hline Wing area & $77 \mathrm{~m}^{2}$ \\
Span & $15.4 \mathrm{~m}$ \\
Aircraft mass & $13.9 \mathrm{t}$ \\
Moment of inertia $I_{x x}$ & $91122 \mathrm{~kg} \mathrm{~m}^{2}$ \\
Moment of inertia $I_{y y}$ & $31560 \mathrm{~kg} \mathrm{~m}^{2}$ \\
Moment of inertia $I_{z z}$ & $122682 \mathrm{~kg} \mathrm{~m}^{2}$ \\
\hline
\end{tabular}

Table 1 Characteristics of UCAV configuration

\subsection{Maneuvers in Wind Tunnel}

During flight tests, the aircraft motion is induced by control surface deflections, which are defined in order to permit an adequate identifiability. In the wind tunnel, the excitation of the model motion is performed directly by the MPM in clean configuration or with fixed control surface deflections. Apart from sinusoidal oscillations at static reference conditions, the MPM can execute arbitrary maneuvers, which are optimized for system identification purposes in this case. The time step does not have to be constant but can be - even within one maneuver definition - adapted to the accelerations which have to be resolved. The smallest possible time step is $10 \mathrm{~ms}$.

For the latest test campaign with the DLR-F19 model, new quasi-steady pitch maneuvers (Fig. 5) with and without superimposed harmonic excitations or frequency sweeps have been designed to significantly reduce the time for the wind tunnel experiments. Furthermore, these maneuvers improve the quality of the aerodynamic dataset generation 
based on the nonlinear system identification approach described in section 3.2. This is due to the fact that measurements are available continuously with varying motion frequencies at each spot of the investigated angle of attack range.
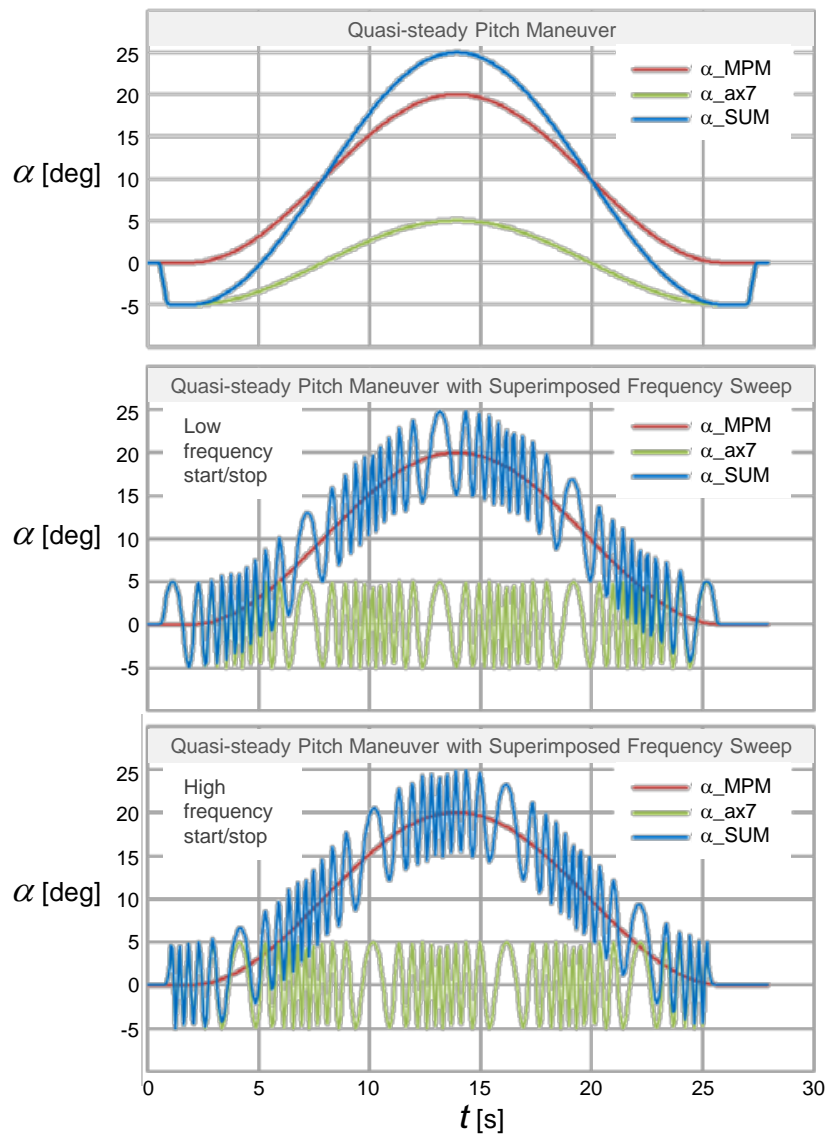

Fig. 5 Time histories of pitch maneuvers used for the motion generation in the wind tunnel and subsequent system identification

The wind tunnel experiments have been conducted at a Mach number of 0.15 and a Reynolds number of $1.6 \cdot 10^{6}$, which was based on a chord reference length of 0.479 . Al maneuvers performed are based on a slow " 1 - cosine" $\alpha$ sweep from $0^{\circ}$ to $20^{\circ}$ generated by the Stewart platform and a superimposed $\alpha$-excitation by the $7^{\text {th }}$ axis with $\pm 5^{\circ}$. The sum of both inputs are leading to the maximum possible pitch range of $\alpha=-5^{\circ}$ to $\alpha=25^{\circ}$. The superimposed $\alpha$-excitation can be an additional quasisteady pitch maneuver (top of Fig. 5) or harmonic oscillations of either constant or varying frequency. The quasi-steady pitch maneuvers with superimposed frequency sweeps are performed in two ways, starting (and stopping) either with the lowest frequency of $0.3 \mathrm{~Hz}$ swelling up to $3 \mathrm{~Hz}$ ( $\alpha$-sweep-up maneuver, middle of Fig. 5), or starting with the highest frequency of $3 \mathrm{~Hz}$ swelling down to $0.3 \mathrm{~Hz}$ ( $\alpha$-sweep-down maneuver, bottom of Fig. 5). The latter two maneuvers are used here to get a suitable frequency distribution over angle of attack. Furthermore, the MPM permits to superimpose motions about other axes, e.g. rolling or yawing oscillations. This has been realized during other test campaigns, but is not shown in the present paper. As already mentioned above, the benefit of the superimposed maneuvers is a significant time saving of expensive wind tunnel time. The achievable saving depends on the test program but can exceed $75 \%$.

\subsection{Data Acquisition}

Data acquisition for all dynamic tests is performed using a data acquisition system with a sampling frequency of $f_{s}=600 \mathrm{~Hz}$. No corrections for wall or blockage effects are applied to the forced motion time history data. As mentioned above, the model's attitude is measured with a pair of video cameras, evaluating the location of markers applied to the model surface at the same sampling frequency. The derivatives are calculated from the internally measured forces and moments. This calculation is based on the assumption that the wind tunnel model is ideally stiff.

When the wind is turned on and the model performs an unsteady motion, the recorded signals always contain mass and initial forces and moments in addition to the aerodynamic forces and moments. For the further data evaluation of the aerodynamic forces and moments, these mass and inertial components have to be eliminated. This can be achieved by performing measurements with the model executing exactly the same oscillation (or maneuver) in wind-off conditions. In case of the Fourier analysis, the wind-off data is subtracted from the wind-on data after the Fourier coefficients (described in [12]) have been calculated. In case of the evaluation by parameter estimation, this subtraction is performed prior to the estimation.

\section{ANALYSIS SET-UP}

\subsection{Classical Approach of Wind Tunnel Data Evaluation}

Classical approaches for the determination of dynamic derivatives on the basis of wind tunnel experiments require that the aerodynamic forces and moments are linear functions of model attitude and angular speed, or, at heave and lateral oscillation, linear functions of translatory speed and acceleration. Several methods for the calculation of dynamic derivatives based on a linear assumption exist [3].

For a pitching oscillation, the Fourier analysis according to [12] yields the pitching moment parameters $C_{m_{0}}, C_{m_{\alpha}}$ and $C_{m_{\dot{\alpha}}}+C_{m_{q}}$ as well as the corresponding parameters for $C_{X}$ and $C_{z}$. The result of a linear analysis, taking only the fundamental frequency into account, is given in [13]. It clearly indicates the inadequate representation of nonlinear aerodynamic data in the case of DLR-F19.

\subsection{System Identification Approach}

Early in 2009, DLR investigated the general applicability of its system identification (SysID) method to dynamic wind tunnel data, also demonstrating that the application of linear aerodynamic models is principally possible but has only limited potential. The applied SysID approach is based on a Matlab/Simulink $\AA$ parameter estimation procedure [14]. An equation error algorithm is used to minimize the differences between model-fixed measured and simulated forces and moments or their respective coefficients. In contrast to the parameter estimation, the SysID comprises the development of the model structure, which is an essential part in case of unconventional aircraft aerodynamics. Whilst in this context, the parameters appearing in the aerodynamic model of the DLR-F19 are estimated with a standardized procedure, 
the model structure has been developed through engineering judgment and reasoning.

Within the system identification application, DLR developed an equivalent nonlinear 6-DoF aerodynamic model, which represents the basis for the parameter estimation and can be used in the same form for real-time flight mechanical simulations later on. Another essential advantage of the 6-DoF approach is the fact that a single set of aerodynamic coefficients/derivatives covers the entire tested angle of attack regime, accounting additionally for aerodynamic cross couplings.

Although a linear approach may give good results for the low a regime of the DLR-F19 model (where the flow is relatively steady), it is likely to be unsuitable for the modeling of more complex effects of turbulent or detached flow at higher angles of attack. To address this, a more general nonlinear model was developed throughout the SysID process to provide the approximation of the aerodynamic total aircraft coefficients without direct modeling of the vortical airflow characteristics (so called equivalent modeling). Finally, this model uses linear parameters but nonlinear formulations e.g. for time delays, quadratic and cubic sideslip effects and high lift hysteresis. Furthermore, all parameters are estimated linearly at distinct $\alpha$-breakpoints and are then summed up to a total nonlinear aerodynamic model as described below.

The six equations are based on a number of angle of attack breakpoints to cope with the nonlinearities in the angle of attack dependencies. In between these breakpoints the corresponding derivative is linearly interpolated. The breakpoints themselves are estimated along with the aerodynamic parameters so as to be automatically concentrated in the areas with significant changes of the angle of attack dependent derivatives. A simple smoothing function is applied to the total coefficients in order to remove excessive peaks, i.e. each data value is adjusted depending on the spacing of the associated angle of attack value to the preceding and succeeding angle of attack breakpoints. The derivatives are linear with respect to the other input signals, which are $\beta, p, q, r$, and angle of attack rate $\dot{\alpha}$. The rates are calculated in a pre-processing step by means of numerical differentiation. In the case of plunge tests, the angle of attack rate $\dot{\alpha}$ is used to avoid high correlations during the estimation of $\dot{\alpha}$ and $q$ derivatives. In all other test cases, the signals of $\dot{\alpha}$ and $q$ are identical.

For DLR-F19 configuration, drag is modeled as a function of lift to the power of 4 - in contrast to the classical formula - scaled by a 'vortex drag factor' $F_{v D}$, which is to be estimated. The use of the power of 4 leads to a significantly better fit in drag, showing a relatively flat dependency on lift at low angles of attack and a steep dependency at high angles of attack. In the longitudinal equations, the high-lift hysteresis formula suggested in [15] and [16] is used in addition, influencing primarily lift, but also drag and pitching moment. Thus, the equivalent aerodynamic model equations are as follows:

$$
\begin{aligned}
\mathrm{C}_{\mathrm{L}}= & \mathrm{C}_{\mathrm{L} 0}+\Delta \mathrm{C}_{\mathrm{L}}^{\alpha}+\Delta \mathrm{C}_{\mathrm{L}}^{\beta}+\Delta \mathrm{C}_{\mathrm{L}}^{(\mathrm{q}+\dot{\alpha})}+\Delta \mathrm{C}_{\mathrm{L}}^{\mathrm{r}}+\Delta \mathrm{C}_{\mathrm{L}}^{\mathrm{p}}+\Delta \mathrm{C}_{\mathrm{L}}^{\dot{\alpha}}+\Delta \mathrm{C}_{\mathrm{L}}^{\mathrm{B}}+\Delta \mathrm{C}_{\mathrm{L}}^{\mathrm{OB}}+\Delta \mathrm{C}_{\mathrm{L}}^{\mathrm{SP}} \\
& +\Delta \mathrm{C}_{\mathrm{L}}^{\text {hys }}, \\
\mathrm{C}_{\mathrm{D}}= & \mathrm{C}_{\mathrm{D} 0}+\Delta \mathrm{C}_{\mathrm{D}}^{\alpha}+\Delta \mathrm{C}_{\mathrm{D}}^{\beta}+\Delta \mathrm{C}_{\mathrm{D}}^{(\mathrm{q}+\dot{\alpha})}+\Delta \mathrm{C}_{\mathrm{D}}^{\mathrm{r}}+\Delta \mathrm{C}_{\mathrm{D}}^{\mathrm{p}}+\Delta \mathrm{C}_{\mathrm{D}}^{\dot{\alpha}}+\Delta \mathrm{C}_{\mathrm{D}}^{\mathrm{B}}+\Delta \mathrm{C}_{\mathrm{D}}^{\mathrm{OB}}+\Delta \mathrm{C}_{\mathrm{D}}^{\mathrm{SP}} \\
& +\Delta \mathrm{C}_{\mathrm{D}}^{\text {hys }}+\mathrm{F}_{\mathrm{VD}} \cdot \mathrm{C}_{\mathrm{L}}^{4},
\end{aligned}
$$

$$
\begin{aligned}
& \mathrm{C}_{\mathrm{Y}}=\mathrm{C}_{\mathrm{Y} O}+\Delta \mathrm{C}_{\mathrm{Y}}^{\alpha}+\Delta \mathrm{C}_{\mathrm{Y}}^{\beta}+\Delta \mathrm{C}_{\mathrm{Y}}^{(\mathrm{q}+\dot{\alpha})}+\Delta \mathrm{C}_{\mathrm{Y}}^{\mathrm{r}}+\Delta \mathrm{C}_{\mathrm{Y}}^{\mathrm{p}}+\Delta \mathrm{C}_{\mathrm{Y}}^{\dot{\alpha}}+\Delta \mathrm{C}_{\mathrm{Y}}^{\mathrm{IB}}+\Delta \mathrm{C}_{\mathrm{Y}}^{\mathrm{OB}}+\Delta \mathrm{C}_{\mathrm{Y}}^{\mathrm{SP}} \text {, } \\
& \mathrm{C}_{\mathrm{l}}=\mathrm{C}_{10}+\Delta \mathrm{C}_{1}^{\alpha}+\Delta \mathrm{C}_{1}^{\beta}+\Delta \mathrm{C}_{1}^{(\mathrm{q}+\dot{\alpha})}+\Delta \mathrm{C}_{1}^{\mathrm{r}}+\Delta \mathrm{C}_{1}^{\mathrm{p}}+\Delta \mathrm{C}_{1}^{\dot{\alpha}}+\Delta \mathrm{C}_{\mathrm{l}}^{\mathrm{B}}+\Delta \mathrm{C}_{\mathrm{l}}^{\mathrm{OB}}+\Delta \mathrm{C}_{\mathrm{l}}^{\mathrm{SP}} \text {, } \\
& \mathrm{C}_{\mathrm{m}}=\mathrm{C}_{\mathrm{m} 0}+\Delta \mathrm{C}_{\mathrm{m}}^{\alpha}+\Delta \mathrm{C}_{\mathrm{m}}^{\beta}+\Delta \mathrm{C}_{\mathrm{m}}^{(\mathrm{q}+\dot{\alpha})}+\Delta \mathrm{C}_{\mathrm{m}}^{\mathrm{r}}+\Delta \mathrm{C}_{\mathrm{m}}^{\mathrm{p}}+\Delta \mathrm{C}_{\mathrm{m}}^{\dot{\alpha}}+\Delta \mathrm{C}_{\mathrm{m}}^{\mathrm{B}}+\Delta \mathrm{C}_{\mathrm{m}}^{\mathrm{OB}}+\Delta \mathrm{C}_{\mathrm{m}}^{\mathrm{SP}} \\
& +\Delta \mathrm{C}_{\mathrm{m}}^{\text {hys }} \text {, } \\
& \mathrm{C}_{\mathrm{n}}=\mathrm{C}_{\mathrm{n} 0}+\Delta \mathrm{C}_{\mathrm{n}}^{\alpha}+\Delta \mathrm{C}_{\mathrm{n}}^{\beta}+\Delta \mathrm{C}_{\mathrm{n}}^{(\mathrm{q}+\dot{\alpha})}+\Delta \mathrm{C}_{\mathrm{n}}^{\mathrm{r}}+\Delta \mathrm{C}_{\mathrm{n}}^{\mathrm{p}}+\Delta \mathrm{C}_{\mathrm{n}}^{\dot{\alpha}}+\Delta \mathrm{C}_{\mathrm{n}}^{\mathrm{B}}+\Delta \mathrm{C}_{\mathrm{n}}^{\mathrm{OB}}+\Delta \mathrm{C}_{\mathrm{n}}^{\mathrm{SP}} \text {, } \\
& \text { with }(i=L, D, Y, I, m, n) \text { : } \\
& \Delta \mathrm{C}_{\mathrm{i}}^{\alpha}=\mathrm{C}_{\mathrm{ia}}(\mathrm{Bp}) \quad \cdot \Delta \mathrm{\alpha}\left(\mathrm{t}-\Delta \mathrm{t}_{\mathrm{a}}\right)
\end{aligned}
$$

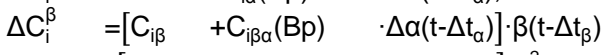

$$
\begin{aligned}
& +\left[\quad+\mathrm{C}_{\mathrm{i} \beta^{2} \alpha}(\mathrm{Bp}) \quad \cdot \Delta \alpha\left(\mathrm{t}-\Delta \mathrm{t}_{\alpha}\right)\right] \cdot \beta^{2}\left(\mathrm{t}-\Delta \mathrm{t}_{\beta}\right) \text { for } i=L, D, m \\
& +\left[\quad+C_{i \beta^{3} \alpha}(\mathrm{Bp}) \quad \cdot \Delta \alpha\left(\mathrm{t}-\Delta \mathrm{t}_{\alpha}\right)\right] \cdot \beta^{3}\left(\mathrm{t}-\Delta \mathrm{t}_{\beta}\right) \text { for } i=Y, l, n, \\
& \Delta \mathrm{C}_{\mathrm{i}}^{(\mathrm{q}+\dot{\alpha})}=\left[\mathrm{C}_{\mathrm{i}(\mathrm{q}+\dot{\alpha})}+\mathrm{C}_{\mathrm{i}(\mathrm{q}+\dot{\alpha}) \mathrm{a}}(\mathrm{Bp}) \cdot \Delta \mathrm{\alpha}\left(\mathrm{t}-\Delta \mathrm{t}_{\alpha}\right)\right] \cdot \mathrm{q}\left(\mathrm{t}-\Delta \mathrm{t}_{\mathrm{q}}\right) \text {, } \\
& \Delta \mathrm{C}_{\mathrm{i}}^{\mathrm{r}}=\left[\mathrm{C}_{\mathrm{ir}} \quad+\mathrm{C}_{\text {ira }}(\mathrm{Bp}) \quad \cdot \Delta \mathrm{\alpha}\left(\mathrm{t}-\Delta \mathrm{t}_{\alpha}\right)\right] \cdot \mathrm{r}\left(\mathrm{t}-\Delta \mathrm{t}_{\mathrm{r}}\right), \\
& \Delta \mathrm{C}_{\mathrm{i}}^{\mathrm{p}}=\left[\begin{array}{lll}
\mathrm{C}_{\mathrm{ip}} & +\mathrm{C}_{\text {ipa }}(\mathrm{Bp}) \quad \cdot \Delta \alpha\left(\mathrm{t}-\Delta \mathrm{t}_{\alpha}\right)
\end{array}\right] \cdot \mathrm{p}\left(\mathrm{t}-\Delta \mathrm{t}_{\mathrm{p}}\right), \\
& \Delta \mathrm{C}_{\mathrm{i}}^{\dot{\alpha}}=\left[\begin{array}{lll}
\mathrm{C}_{\mathrm{i} \dot{\alpha}} & +\mathrm{C}_{\mathrm{i} \alpha}(\mathrm{Bp}) \quad \cdot \Delta \mathrm{\alpha}\left(\mathrm{t}-\Delta \mathrm{t}_{\alpha}\right)
\end{array}\right] \cdot\left(\dot{\mathrm{\alpha}}\left(\mathrm{t}-\Delta \mathrm{t}_{\dot{\alpha}}\right)-\mathrm{q}\left(\mathrm{t}-\Delta \mathrm{t}_{\mathrm{q}}\right)\right), \\
& \Delta \mathrm{C}_{\mathrm{i}}^{\mathrm{IB}}=\left[\mathrm{C}_{\mathrm{iLIB}}+\mathrm{C}_{\mathrm{iLIB \alpha}}(\mathrm{Bp}) \cdot \Delta \mathrm{\alpha}\left(\mathrm{t}-\Delta \mathrm{t}_{\alpha}\right)\right] \cdot \mathrm{LIB}, \\
& +\left[\mathrm{C}_{\mathrm{iRIB}}+\mathrm{C}_{\mathrm{iRIBa}}(\mathrm{Bp}) \cdot \Delta \alpha\left(\mathrm{t}-\Delta \mathrm{t}_{\alpha}\right)\right] \cdot \mathrm{RIB}, \\
& \Delta \mathrm{C}_{\mathrm{i}}^{\mathrm{OB}}=\left[\mathrm{C}_{\mathrm{iLOB}}+\mathrm{C}_{\mathrm{iLOBa}}(\mathrm{Bp}) \cdot \Delta \mathrm{\alpha}\left(\mathrm{t}-\Delta \mathrm{t}_{\mathrm{a}}\right)\right] \cdot \mathrm{LOB} \\
& +\left[\mathrm{C}_{\mathrm{iROB}}+\mathrm{C}_{\mathrm{iROB \alpha}}\left(\mathrm{Bp} \cdot \Delta \mathrm{\alpha}\left(\mathrm{t}-\Delta \mathrm{t}_{\mathrm{\alpha}}\right)\right] \cdot \mathrm{ROB},\right. \\
& \Delta \mathrm{C}_{\mathrm{i}}^{\mathrm{SP}}=\left[\mathrm{C}_{\mathrm{iSP} 20}+\mathrm{C}_{\mathrm{iSP} 20 \mathrm{\alpha}}(\mathrm{Bp}) \cdot \Delta \mathrm{\alpha}\left(\mathrm{t}-\Delta \mathrm{t}_{\alpha}\right)\right] \cdot \mathrm{SP} 20 \text {, } \\
& +\left[\mathrm{C}_{\text {iSP25 }}+\mathrm{C}_{\text {iSP25a }}(\mathrm{Bp}) \cdot \Delta \mathrm{\alpha}\left(\mathrm{t}-\Delta \mathrm{t}_{\mathrm{\alpha}}\right)\right] \cdot \mathrm{SP} 25, \\
& +\left[\mathrm{C}_{\text {iSP20_1B }} \cdot \mathrm{SP} 20+\mathrm{C}_{\text {iSP25_1B }} \cdot \mathrm{SP} 25\right] \cdot \text { LIB. }
\end{aligned}
$$

The notation $\Delta C_{i}^{j}$ denotes a delta coefficient of the force or moment $i$, whose strongest influence is the parameter $j$. Most of the coefficients consist of a constant part and parameter values at each breakpoint (Bp). The latter are valid above the corresponding angle of attack breakpoint, i.e., each parameter value gives the slope between the current and the next breakpoint. This derivative at the breakpoint is then multiplied by $\Delta \alpha$, which is the difference between the current angle of attack and the angle of attack at the current breakpoint. Thus, a total a slope is accumulated to represent a quasi-steady coefficient trajectory (which is - in contrast to linear methods - not exactly the trajectory of the steady-state experiments, however close to it). The hysteresis loops are then added to this quasi-steady coefficient using equivalent time delays, $\mathrm{k}\left(\mathrm{t}-\Delta \mathrm{t}_{\mathrm{k}}\right)$, in $\alpha, \beta, \quad p, q, \quad r$ and $\dot{\alpha}$ to roughly approximate the non-stationary changes in the vortex formations. The control surfaces LIB, RIB, LOB, ROB, SP20 and SP25 are fixed to certain deflections (zero for clean configuration), thus excluding additional time depending effects. Left and right control surfaces show slightly different (asymmetric) efficiencies and are consequently estimated separately. The labels SP20 and SP25 denote $20 \%$ and $25 \%$ split flaps depth. In the wind tunnel experiments either spilt flaps with $20 \%$ or $25 \%$ depth were used. The parameter estimation considers the corresponding parameters in each case. To cope with different flap efficiencies in case of separately or simultaneously deflected inboard control surfaces and split flaps, a constant term was introduced and estimated. For now, this term could be estimated only for the left inboard control surface because of a lag of corresponding experiments for the right flap.

The high-lift hysteresis formula for parameter estimation purposes suggested in [15] and [16] is a hyperbolic function depending on the angle of attack and is often used to model the stall characteristics of conventional airplanes due to partial airflow separation: 
$X=\frac{1}{2}\left[1-\tanh \left(\mathrm{a}_{1}\left(\alpha\left(\mathrm{t}-\Delta \mathrm{t}_{\alpha}\right)-\mathrm{T} \cdot \dot{\mathrm{\alpha}}\left(\mathrm{t}-\Delta \mathrm{t}_{\alpha}\right)-\mathrm{\alpha}^{*}\right)\right)\right]$,

$\Delta C_{L}^{\text {hys }}=C_{L \alpha}\left[\frac{1}{4}(1+\sqrt{X})^{2}-1\right] \alpha$,

$\Delta \mathrm{C}_{\mathrm{D}}^{\text {hys }}=\mathrm{C}_{\mathrm{DX}} \cdot \mathrm{X}$,

$\Delta \mathrm{C}_{\mathrm{m}}^{\text {hys }}=\mathrm{C}_{\mathrm{mX}} \cdot \mathrm{X}$,

with $a_{1}, \alpha^{*}$ and $t$, as well as $C_{D x}$ and $C_{m x}$ to be estimated.

Here, $a_{1}$ characterizes the reduction in slope of the lift curve, $\alpha^{*}$ represents the angle of attack where half of airflow is detached, and $t$ is the hysteresis time constant. The non-dimensional parameter $X$ corresponds to the flow separation point along the chord and can range from 1 for fully attached to 0 for fully separated flow. $C_{D x}$ and $C_{m x}$ are hysteresis influence factors on drag and pitching moment respectively.

\subsection{VSAERO Aerodynamics}

As an example for fast and simple aerodynamic tools, the commercial VSAERO code [4] is used to get an alternative aerodynamic dataset of the present UCAV configuration. VSAERO is a 3D singularity method based on inviscid and incompressible potential flow theory, calculated on surface meshes. It computes the aerodynamic force and moment coefficients typically within a few seconds of computation time. For investigating compressible flows, several compressibility corrections are included; viscous drag can optionally be considered by an iteratively coupled boundary layer module. For damping derivative computation, quasi-steady rotations can be applied.

In the analysis presented here, VSAERO is applied on the basis of the CPACS file (cf. section 1) of the UCAV configuration. As flow conditions, a range of Mach and Reynolds numbers as well as the angles of attack and sideslip are specified. Control surface deflections are implemented by rotation of the normal vectors of the corresponding panels around the hinge line. The option to apply viscous drag in VSAERO is not used here; instead a simple formula based on flat plate analogy is employed.

VSAERO is a well-proven tool for conventional transport aircraft. However, due to the limitations of the underlying model, it is obvious that significant vortex and separation dominated effects of such a highly swept configuration especially at higher angles of attack - cannot be modeled correctly. A detailed analysis of this aspect can be found in [1]. The question in this paper is whether a rather simple tool like VSAERO, which is developed for inviscid, incompressible flow, can already provide a reasonable first impression of the behavior and critical properties of such a configuration. Figure 6 shows a surface and wake mesh of the DLR-F19 UCAV configuration for the use with VSAERO.

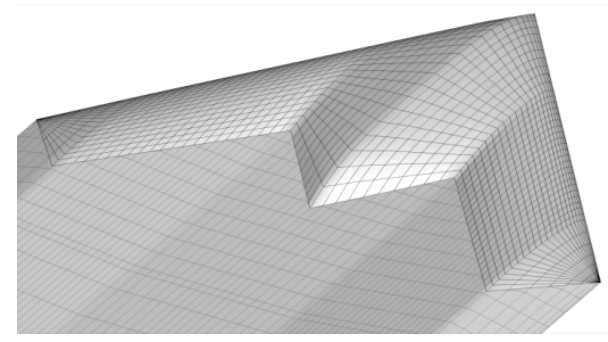

Fig. 6 VSAERO mesh of the DLR-F19 UCAV configuration

\subsection{Flying Qualities Analysis}

The flying qualities analysis shall provide first insights of the flight dynamic behavior of the UCAV configuration. The analysis focuses on comparing the dynamic behavior of the previously determined model of the configuration derived from wind tunnel data (cf. section 3.2) with the dynamics of the same aircraft configuration containing the aerodynamic dataset computed with the aerodynamic tool VSAERO (cf. section 3.3). In order to analyze and compare the flight dynamics of the overall aircraft, the aerodynamic models obtained from system identification and VSAERO are integrated into a 6-degrees-of-freedom aircraft simulation environment. This flight dynamics model and the framework of the flying qualities analysis will be briefly described in the following.

\subsubsection{Flight Dynamics Model}

The flight dynamics model is developed in an environment called flightSim, which consists of:

a model integration process that allows for fully automatic generation of aircraft flight dynamics models from CPACS or other databases. This process is part of a standardized Dynamic Aircraft Model Integration Process (DAMIP) developed at the DLR Institute of System Dynamics and Control [17],

- a Modelica ${ }^{\circledR}$-based library of aircraft models and model components that allows for automatic generation of dedicated runtime models (6-DoF, 3DoF, forward, inverse, open loop, closed loop) for various types of model analyses (flight dynamics, performance, mission simulation, etc.), see [18] for more details.

In order to generate a flight dynamics model on the basis of the aerodynamic dataset computed by VSAERO, the standard process as applied in several DLR projects and described in [19] was used. The aerodynamic dataset is thereby provided in the form of a four-dimensional performance map of the aerodynamic force and moment coefficients of the clean configuration, depending on Mach number, Reynolds number, and angles of attack and sideslip. The effects resulting from each control surface deflection as well as the damping derivatives are provided as delta-performance maps, which have to be added to the values of the clean configuration. For inclusion of the aerodynamics model obtained by means of system identification applied to the wind tunnel measurements, a dedicated aerodynamics module was developed. The model structure resulting from the system identification process is thereby directly used in the flight dynamics model. Both simulation models are based on nonlinear Newton-Euler equations of motion for a rigid body and use the same weight and balance, systems, sensors, and engine modules. The engine modules are based on engine performance maps, which are described in detail in [20].

The simulation models feature standardized inputs (control deflections, engine throttle settings, combined control surface deflections for trimming, wind components) and outputs (states, air data sensors, inertial sensors, navigation sensors, etc.), as well as scripts for trimming and linearization. For the flying qualities analysis presented here, not all modules of the flight dynamics model are necessarily required. Components like air data or navigation sensors, for instance, are not needed for this 
analysis. Their integration in the model, however, allows a general use of the flight dynamics model for further assessments of the aircraft behavior.

\subsubsection{Framework of Flying Qualities Analysis}

The main purpose of flying qualities criteria is to describe the controllability of an air vehicle via the evaluation of the eigenvalues. Available flying qualities criteria are derived from the point of controllability via a human pilot. For UAVs these criteria can be considered as applicable as well, nevertheless, given boundaries for various levels of flying qualities need to be revisited. The flying qualities criteria are thus applied here to get a general, qualitative impression of the flight dynamic behavior of the considered aircraft and to accomplish the intended comparison of the behavior obtained with the two different aerodynamic datasets.

The flying qualities analysis is performed with the analysis tool HAREM (HAndling qualities REasearch using Matlab) [21], [22]. This tool was developed at the Institute of Flight Systems of DLR and allows the assessment of a wide range of handling and flying qualities criteria in an automatic manner. It applies the criteria to linear flight dynamics aircraft models and automatically delivers the corresponding flying qualities level as well as figures showing the criterion graphs. For the presented results HAREM has been integrated into a workflow, in which the tool flightSim (cf. section 3.4.1) generates a flight dynamics model of the UCAV configuration and passes this to HAREM as a CPACS file. A detailed description of the application of HAREM in combination with CPACS is given in [23].

For the application of the flying qualities criteria of HAREM the flight phase and aircraft category of the considered configuration have to be defined according to the specifications of MIL-STD-1797 [24]. The flight phase is defined by the conditions of the wind tunnel experiments. As the experiments have been performed at a Mach number of 0.15 , the resulting aerodynamic dataset is valid for the low-speed range only. These airspeeds occur only during takeoff, approach and landing. The corresponding flight phase category C of MIL-STD-1797 is thus considered for the analysis. The flight condition underlying the present flying qualities analysis is a trimmed horizonta flight at $500 \mathrm{~m}$ altitude with a calibrated airspeed of $100 \mathrm{~m} / \mathrm{s}$, which corresponds to a Mach number of 0.3 . At this Mach number compressibility effects are still negligible such that the aerodynamic dataset determined by low speed wind tunnel experiments is still valid. Both aircraft classes II and IV are considered in the analysis. Class II is considered because the currently specified mission of the UCAV configuration contains comparably moderate maneuvers for a fighter aircraft. This correlates to the definition of aircraft class II for medium weight aircraft with low to medium maneuverability. For future applications, however, the UCAV configuration may be used as a highly agile fighter aircraft. The flying qualities are thus also assessed for aircraft class IV covering high maneuverability aircraft.

The flying qualities level used here correspond to the definitions of [24]. Level 1 denotes "Satisfactory" flying qualities, level 2 means the flying qualities are "Acceptable", and level 3 describes "Controllable" flying qualities. As already mentioned, the exact flying qualities level is not relevant here because an unmanned configuration is considered. The analysis shall only give a qualitative impression of the dynamic behavior of the UCAV and show the comparison of the two aircraft models using aerodynamic datasets derived with different methods.

\section{RESULTS}

\subsection{System Identification Results}

For the model structure presented in section 3.2 the parameters have been estimated on the basis of the wind tunnel measurements during the quasi-steady and dynamic maneuvers. The results are presented in Fig. 7 to 11 .

Figure 7 shows the time history fit of the DLR-F19 longitudinal and lateral aerodynamic coefficients for a pitch axis excitation, comparing the wind tunnel data and the output of the model developed by means of system identification. The first time slice contains the slow quasisteady pitch maneuver, the second time slice the $\alpha$ sweep-up maneuver and the third time slice the $\alpha$-sweepdown maneuver (cf. Fig. 5 from top to bottom). The latter two superimposed sweep maneuvers are complementing each other generating different combinations of angle of attack amplitude and frequency. For example, the second time slice shows a slow frequency at maximum angle of attack whereas the third time slice has a relatively high frequency at this point. The time histories clearly show the nonlinear behavior of the coefficients. Except for some unsteady aerodynamic effects at high angle of attack, the model fit is very good, simulating the essential nonlinearities. The lateral forces and moments are naturally small during pure pitch maneuvers but at high angles of attack they show some fluctuations due to small asymmetric instabilities in the vortex system. Tiny, unavoidable wind tunnel model inaccuracies between left and right wing may trigger the peaks (being clearly visible during the pure quasi-steady pitch maneuver) where the vortex system alters significantly (cf. Fig. 8).

Figure 8 shows the cross-plot fit of the pitching moment coefficient in clean configuration, in this case of the quasisteady pitch maneuver (first time slice of Fig. 7). The pitching moment coefficient exhibits strong nonlinearities due to vortex effects including e.g. movements of the tip vortex onset point and vortex breakdown. The large influences on the pitching moment due to the vortex systems developing over the highly swept flying wing configuration are discussed in great detail in [25].

The cross-plot includes the wind tunnel data with certain fluctuations at high angles of attack, the dynamic model output and the smooth curve of the quasi-steady portion of the model output. The dynamic model output is based on the complete equations of section 3.2 whereas the quasisteady portion of the model output excludes rates and time delays. Although the pitch rate is intentionally small (cf. Fig. 7), there remain hysteresis effects at high angles of attack due to changes in the vortex system, being visible in both, wind tunnel data and dynamic model output. Correspondingly to Fig. 7, the unsteady aerodynamic impacts increase with rising angle of attack. The essential nonlinear effects are, however, properly replicated 


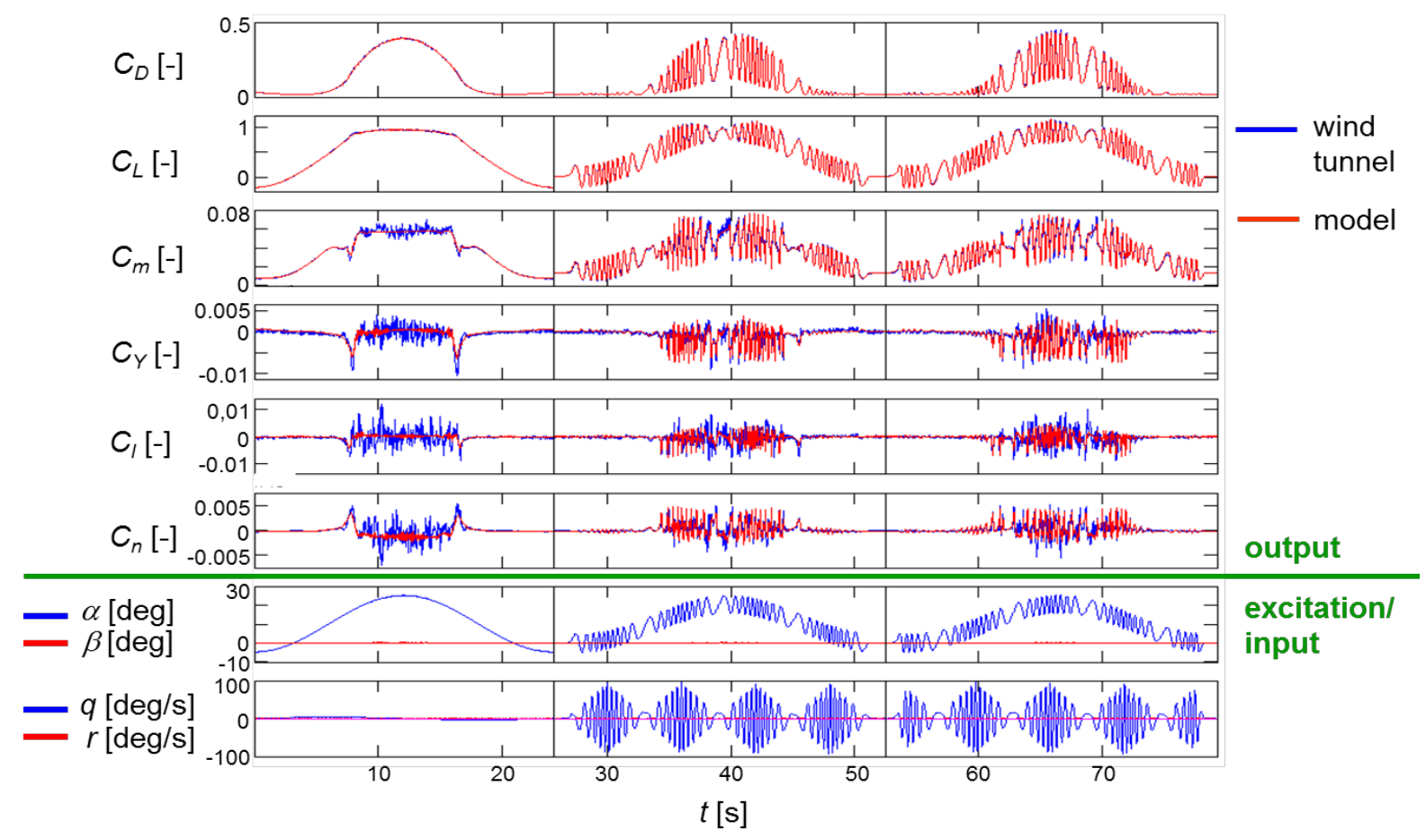

Fig. 7 Time history fit of longitudinal quasi-steady maneuver and $\alpha$-frequency sweeps up and down

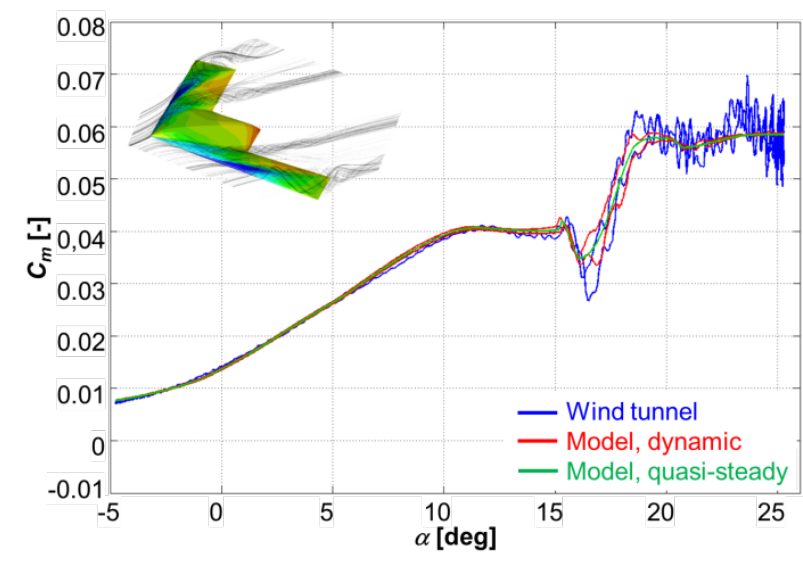

Fig. 8 Cross-plot fit of pitching moment coefficient for the quasi-steady pitch maneuver in clean configuration

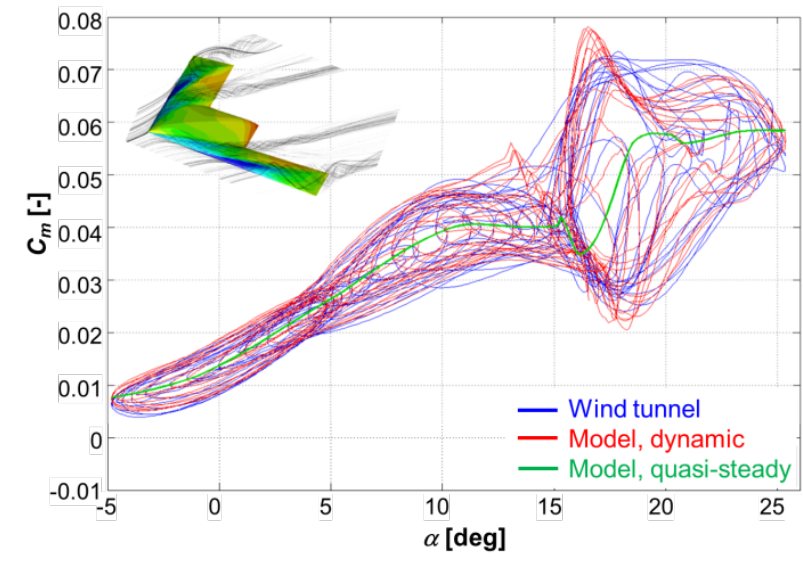

Fig. 9 Cross-plot fit of pitching moment coefficient for the $\alpha$-sweep-down maneuver in clean configuration
Figure 9 shows the cross-plot fit of the pitching coefficient for the $\alpha$-sweep-down maneuver (last time slice of Fig. 7), again for clean configuration. In this case the hysteresis effects due to changes in the vortex system are significant even though the quasi-steady portion of the model output is identical to Fig. 8 (all tests were evaluated in a single identification run). The hysteresis loops of the wind tunnel data and the dynamic model output show similar characteristics and have almost the same envelope.

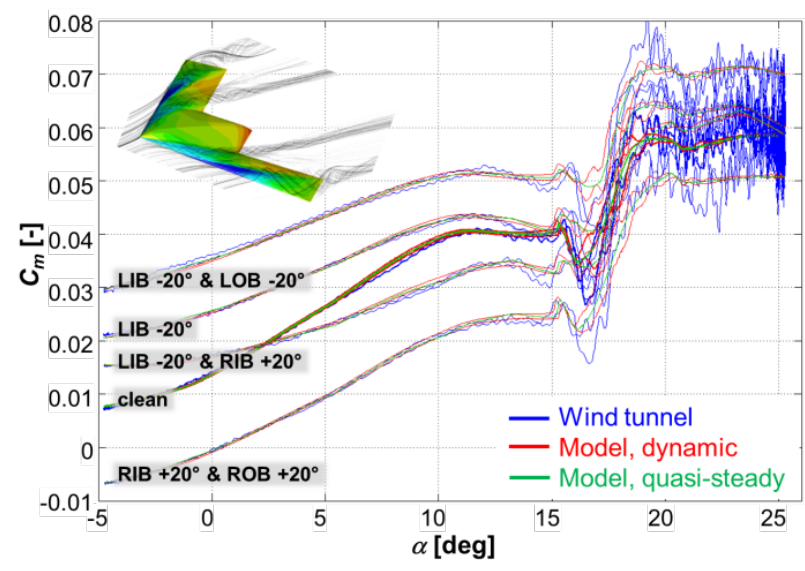

Fig. 10 Cross-plot fit of pitching moment coefficient for quasi-steady pitch maneuvers in particular control surface configurations

The cross-plot fit of the pitching moment coefficient for quasi-steady maneuvers in particular flap configurations is presented in Fig. 10. The trialing edge control surfaces are deflected in different combinations as indicated in the figure: both left trailing edges deflected $20^{\circ}$ upwards (i.e. negative), the left inboard control surface deflected $20^{\circ}$ upwards, left inboard control surface $20^{\circ}$ upwards and right inboard control surface $20^{\circ}$ downwards, no control surface deflection (i.e. clean), and both right control 
surfaces $20^{\circ}$ downwards. The different trailing edge control surface settings do not only cause a vertical curve shift but also lead to a change in the curve slope. Based on Fig. 7 to 10 , the match of the dynamic model output and the wind tunnel data can be considered as adequate, and the extracted aerodynamic model is therefor considered to be suitable for the subsequent flying qualities analysis.

The difference in the pitching moment coefficient for two different control surface deflections provides the control surface efficiency of the corresponding flap. The finally identified DLR-F19 control surface efficiencies are plotted against angle of attack in Fig. 11, in this case for all force and moment coefficients. The labels SP25 and SP20 denote $25 \%$ and $20 \%$ split flaps depth (cf. section 2.2.1). Figure 11 illustrates the strong dependency of the aerodynamic coefficients on angle of attack with exceptionally nonlinear behavior at moderate and high angles of attack.
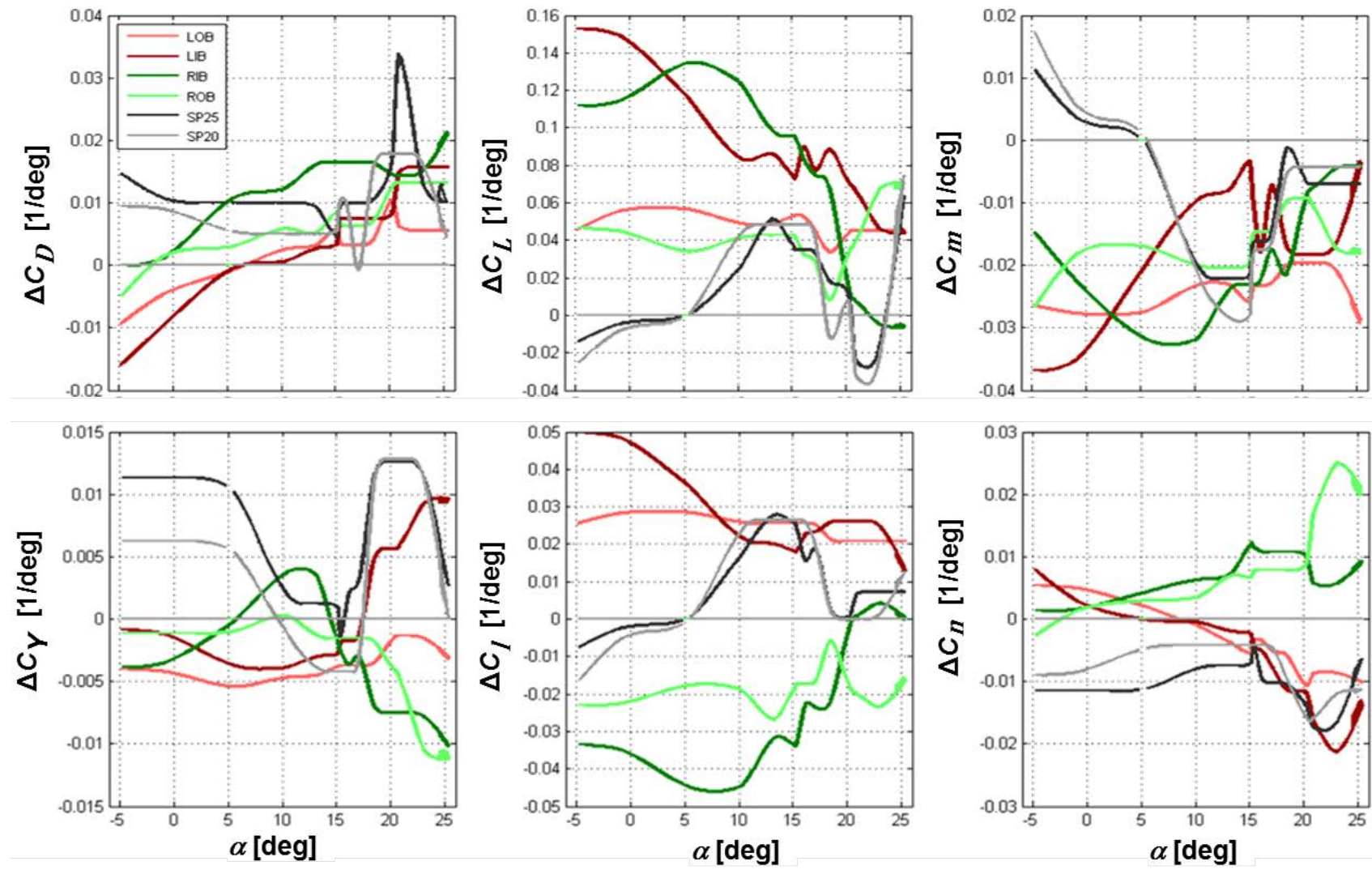

Fig. 11 Determined DLR-F19 control surface efficiencies for the left and right inboard (LIB,RIB) and left and right outboard (LIB,LOB) control surfaces as well as split flaps with 20\% (SP20) and 25\% (SP25) split flap depth

\subsection{Results of VSAERO Analysis}

In contrast to the aerodynamic model resulting from the system identification process, the VSAERO analysis delivers a performance map of the force and moment coefficients. Figure 12 exemplarily shows lift force and pitching moment coefficients versus angle of attack for experimental (i.e. static wind tunnel investigations) and VSAERO results. It can be clearly seen, that the experimental curves show a significant change in their gradients around $10^{\circ}-12^{\circ}$ angle of attack, where the formation of vortices begins. VSAERO, on the other hand, cannot predict such an effect with its underlying potential flow model. However, the difference in pitching moment coefficient must not be overestimated since the chosen moment reference point lies very close to the neutral point. This means, that a small error in neutral point prediction leads to big deviations in the gradient of the pitching moment. Beyond an angle of attack of roughly $15^{\circ}$, strong nonlinearities in the pitching moment curve occur. Thus, this region is not considered for comparison, here.

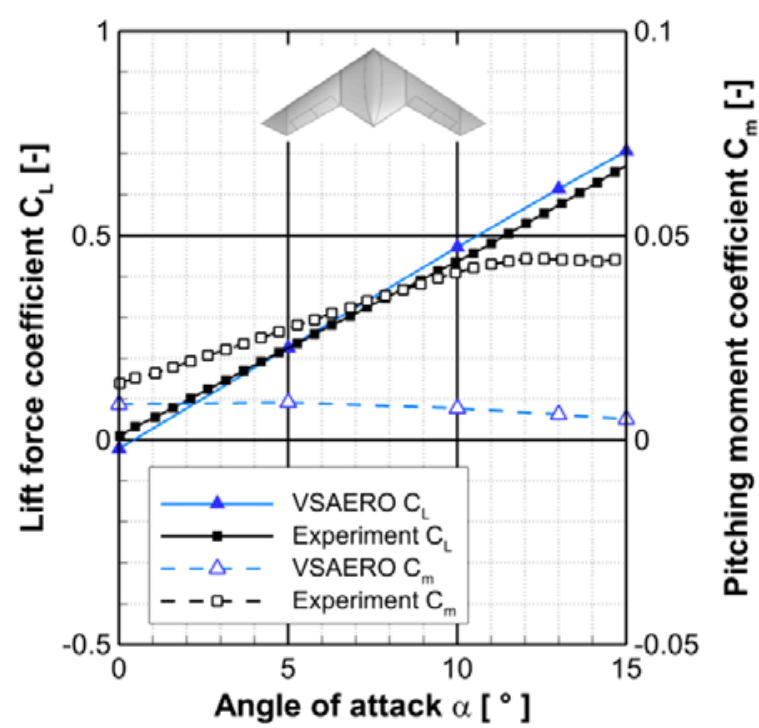

Fig. 12 Comparison of lift force and pitching moment coefficients for experimental and VSAERO results 
A direct comparison of the aerodynamic parameters extracted from static wind tunnel experiments and VSAERO can be found in [2] and [26]. The focus of this paper is, however, to analyze the effects of the two different aerodynamic models (one based on VSAERO and the other one based on dynamic wind tunnel tests and subsequent system identification) on the resulting flying qualities.

\subsection{Evaluation of Flying Qualities}

According to the flying qualities criteria the flying qualities for the longitudinal and lateral axis are analyzed separately and the results are presented in the following sections.

\subsubsection{Longitudinal Dynamics}

The UCAV configuration was designed to be stable in the longitudinal axis. The two aerodynamic datasets yield, however, different static margins. In case of the VSAERO dataset the static margin is $9.8 \%$ of the mean aerodynamic chord, whereas the model derived from the wind tunnel data has a static margin of $3.2 \%$. The center of gravity is identical for both models, but the VSAERO computations deliver an aerodynamic neutral point that is located significantly further aft compared to the neutra point position derived from the wind tunnel measurements. The absolute deviation between the neutral point positions is relatively small. Nevertheless, the resulting effect for the stability margin is significant as the neutral point positions are located very closely to the center of gravity of the aircraft, which is typically the case for a tailless aircraft. The same trend has been found when comparing the VSAERO results with high-fidelity CFD results [2].

The larger static margin resulting from the VSAERO computations also leads to a larger absolute value of the angle-of-attack-dependent pitching moment derivative $C_{m a}$ and consequently to a higher natural frequency of the short period dynamics of the aircraft. The aircraft model based on the VSAERO results has a $C_{m a}$ of -0.30 , whereas the $C_{m a}$ of the aircraft model derived from the wind tunnel data is -0.08 . The resulting higher natural frequency of the VSAERO based model effects a higher agility in the longitudinal axis compared to the aircraft model with aerodynamic data from the wind tunnel experiment. This effect is visible in the criterion graph of the Control Anticipation Parameter (CAP) illustrated in Fig. 13. The parameter $n_{z} / \alpha$ is thereby derived from the steady state response after an elevator step command.

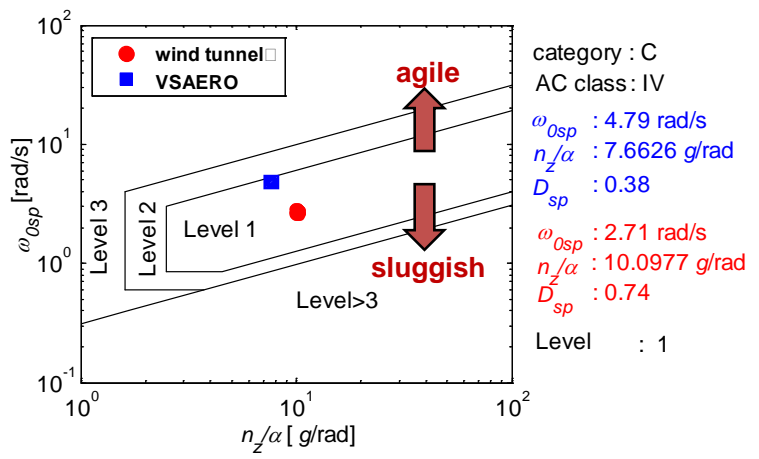

Fig. 13 Comparison of the flying qualities levels of the CAP criterion achieved for the two aircraft models with different aerodynamic datasets
The CAP criterion assesses the initial pitch acceleration in relation to a steady change of the vertical load factor. If this ratio is very large, the aircraft is very agile in the longitudinal axis. It can be noticed in Fig. 13 that, even though the VSAERO based model is located at higher natural frequencies in the CAP criterion graph, it is still placed in the Level 1 region. The damping ratio of the short period mode is lower for the model based on VSAERO computations than for the aircraft model determined from the dynamic wind tunnel experiments. This is another indicator that the VSAERO based aircraft model exhibits more agile dynamics in the longitudinal axis.

A same tendency is visible in the criterion graph of the $C^{*}$ criterion (Fig. 14). The $C^{*}$ criterion [27] specifies requirements for the maximum allowable overshoot in the time response of the parameter $C^{*}$ after a step input in the pitch axis. The $C^{*}$ parameter is defined as a combination of the vertical load factor at the pilot seat and the pitch rate of the aircraft:

$$
C^{*}=\left(n_{z, \text { pilotseat }}+\frac{1}{g} 240 \mathrm{kt} \cdot q\right) .
$$

The factor of $240 \mathrm{kt}$ represents the so-called crossover speed, at which the load factor and pitch rate component of $C^{*}$ are equally weighted. The parameter $n_{z \text {,pilotseat }}$ corresponds to the vertical load factor at the position of the pilot seat. As there is no pilot in the UCAV configuration the vertical load factor at the center of gravity is considered here instead. The $C^{*}$ criterion is usually applied for civil, manned aviation. Nevertheless, it also gives a very good impression of the agility of an unmanned aircraft in the longitudinal axis.

Figure 14 shows that the VSAERO based aircraft model has a strong overshoot in the $C^{*}$ response and is too agile. This is not very critical as the badly damped, too agile $C^{*}$ response can easily be improved by an appropriate flight control system. The important observation derived from Figure 14 is the fact that the $C^{*}$ response of the two models with different aerodynamic datasets is significantly different. This is important to note during the aircraft design process. If only the low-fidelity aerodynamic method were applied this would give the impression of a much more agile aircraft than it might be the case in reality.

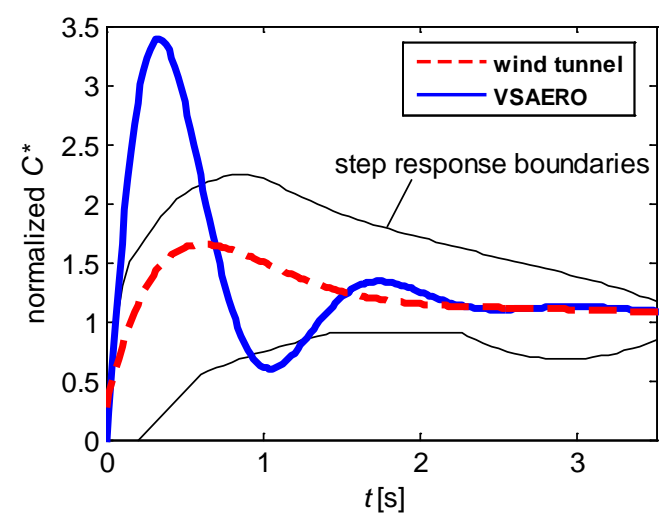

Fig. 14 Comparison of the flying qualities levels of the $C^{*}$ criterion for the two aircraft models with different aerodynamic datasets 


\subsubsection{Lateral Dynamics}

The comparison of the eigenvalues of the lateraldirectional motion in Fig. 15 already shows that the different aerodynamic methods also lead to different lateral-directional dynamics.

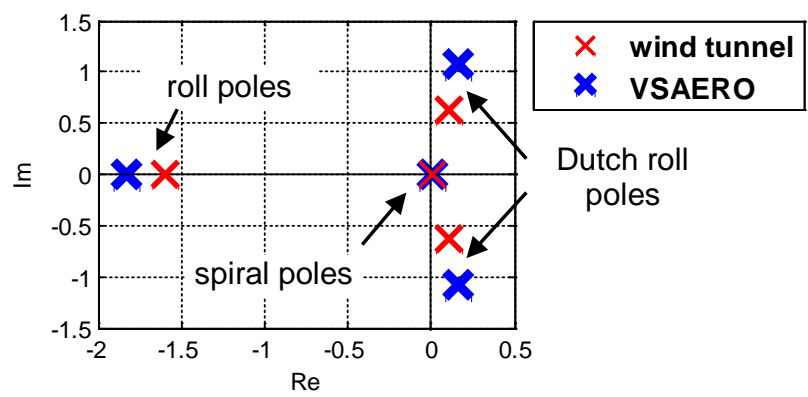

Fig. 15 Eigenvalues of the lateral-directional motion for the two aircraft models with different aerodynamic datasets

The spiral poles are located comparably close to each other, but especially for the roll and Dutch roll poles the difference of the two aerodynamic methods is clearly visible. Nevertheless, both aircraft models achieve the same flying qualities level concerning the different criteria for the lateral-directional eigenmodes. Table 2 shows that the roll time constant fulfills the requirements for level 1 flying qualities in both cases. However, the aircraft model containing the VSAERO dataset exhibits a better value of the roll time constant. At this point, the slightly smaller roll time constant results from the fact that the VSAERO dataset exhibits a larger absolute value of the roll damping derivative $C_{l p}$, which is inversely proportional to the roll time constant. The higher roll damping can be explained by the fact that VSAERO overestimates the lift curve slope, which has also been shown in [2]. As the VSAERO computations are based on inviscid and incompressible potential flow theory, they cannot properly model the complex flow field of a configuration with highly swept leading and trailing edges.

\begin{tabular}{l|c} 
& Roll time constant \\
\hline Maximum value for level 1 & $1 \mathrm{~s}$ \\
VSAERO dataset & $0.57 \mathrm{~s}$
\end{tabular}

\section{Wind tunnel dataset}

$0.67 \mathrm{~s}$

Table 2 Roll time constants for the two aircraft models with different aerodynamic datasets

The spiral mode is unstable for both aerodynamic datasets, but the instability is only small and the requirements for the time to double of $12 \mathrm{~s}$ for level 1 flying qualities can easily be fulfilled, as shown in Table 3. As in the case of the roll time constant, the VSAERO approach delivers slightly better flying qualities. The reason for the difference in the times-to-double of the two aircraft models still needs to be investigated in detail. The consequences are, however, small because the margin to the given threshold value of $12 \mathrm{~s}$ is large in both cases.

\begin{tabular}{l|c} 
& Time to double \\
\hline Minimum value for level 1 & $12 \mathrm{~s}$ \\
VSAERO dataset & $131 \mathrm{~s}$ \\
Wind tunnel dataset & $54 \mathrm{~s}$
\end{tabular}

Table 3 Time to double amplitude of the spiral motion for the two aircraft models with different aerodynamic datasets

The criterion graph for the Dutch roll mode is shown in Fig. 16. For both aerodynamic datasets the UCAV configuration exhibits Dutch roll dynamics corresponding to flying qualities worse than level 3 because the configuration has a negative damping and is dynamically unstable. Moreover, the natural frequency of the Dutch roll is very low for both aerodynamic datasets. In case of the VSAERO based model, the natural frequency is slightly higher because the yaw moment derivative with respect to the sideslip angle is slightly larger, as also demonstrated in [2]. The unfavorable Dutch roll dynamics were expected because the considered UCAV configuration neither possesses a stabilizing vertical tail nor any kind of fins. Concerning the unstable Dutch roll motion the relevant question is whether a flight control system might be able to stabilize the dynamics in the lateral-directional axes. This depends on the availability of sufficient control surface effectiveness and can be assessed only with the closedloop model of the aircraft, which is, however, out of the scope of the paper.

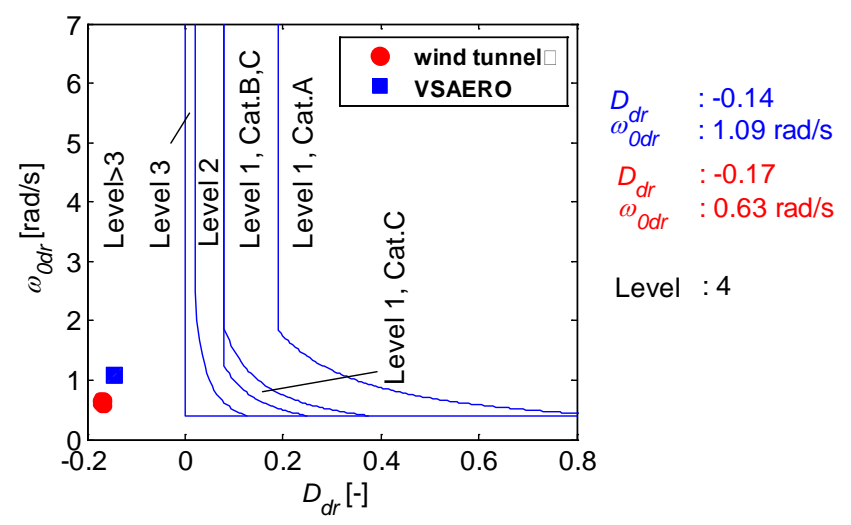

Fig. 16 Comparison of the flying qualities levels of the Dutch roll characteristics for the two aircraft models with different aerodynamic datasets

The requirements for the roll performance as specified by [24] are illustrated in Fig. 17. It shows the time response of the two aircraft models with different aerodynamic datasets for maximum roll control input. The considered UCAV configuration has two trailing edge control surfaces on each wing. In the present case only the outer control surfaces are used for roll control. 


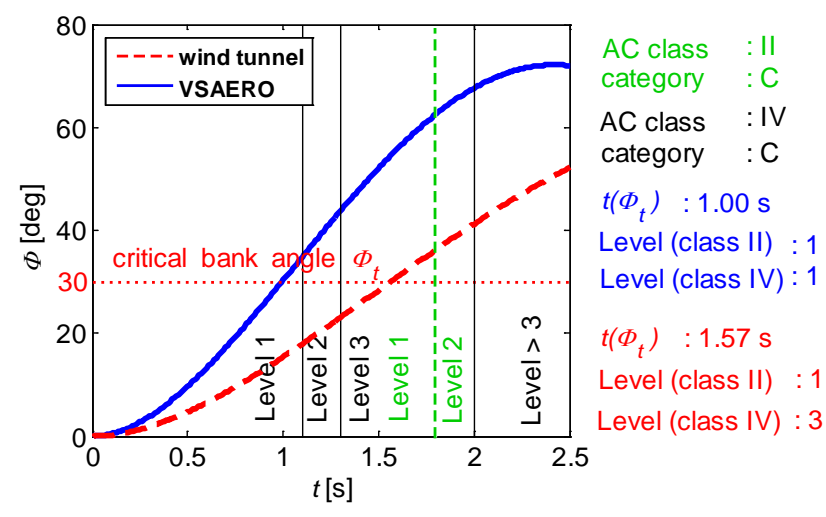

Fig. 17 Comparison of the flying qualities levels of the roll performance for the two aircraft models with different aerodynamic datasets

Depending on the flight phase category and aircraft class, the military standard [24] defines time periods in which specific bank angle values have to be reached. In flight phase category $\mathrm{C}$, a class II aircraft (land-based) has to be able to reach a bank angle of $30^{\circ}$ within $1.8 \mathrm{~s}$ to be classified as level 1 . The corresponding time limit is marked with a dashed vertical line in Fig. 17. If the aircraft is considered as a high maneuverability aircraft of class IV it has to reach a $30^{\circ}$ bank angle in $1.1 \mathrm{~s}$ for level 1 flying qualities. The corresponding time limits for class IV aircraft are shown in black in Fig. 17.

The comparison of bank angle responses of the VSAERO and wind tunnel experiment based aircraft models shows that the VSAERO based model is once again much more agile and has a significantly better roll performance. If the UCAV is evaluated as a class II aircraft, the wind tunnel based model still fulfills level 1 requirement. If the UCAV is to be used as an agile fighter aircraft of class IV, however, the roll performance is too low and only corresponds to level 3. The VSAERO based model, in contrast, reaches level 1 flying qualities as a class IV aircraft as well. The reason for the worse roll performance of the aircraft model derived from the wind tunnel data is the fact that the roll effectiveness was detected to be lower in the wind tunnel experiment than it was predicted by the VSAERO method. The VSAERO method delivered a roll control dependent roll moment derivative $C_{l \text {,ail }}$ of -0.14 whereas the wind tunnel experiment provided a $C_{\text {l,ail }}$ of -0.06 . The overestimation of the efficiency of the control surfaces by VSAERO was expected because the potential flow theory based computation method assumes attached flow and thus predicts full efficiency of the control surfaces. In reality the control surface efficiency is significantly reduced due to flow separation and vortex effects. Moreover, the way how the aerodynamic performance map is stored in CPACS is based on linear superposition of separate control surfaces. This method neglects the crossinfluences between different control surfaces, which is a valid approach for conventional transport aircraft. As shown in [28] however, these effects are not negligible for the considered UCAV configuration. A detailed comparison of the VSAERO results with high-fidelity CDF computations and static wind tunnel experiments can be found in [2], as already mentioned above.

The fact that the roll performance determined on the basis of the dynamic wind tunnel experiments is much smaller could be a critical aspect for the UCAV design, especially if high roll maneuverability is desired for the later application of the UCAV. It has to be kept in mind, however, that only the outer trailing edge control surfaces on the wing are used for roll control here. Even though the inner trailing edge control surfaces are less effective due to their smaller lever arms, an increased roll performance could be achieved by deflecting them simultaneously with the outer control surfaces. It should thus be considered to use all trailing edge control surfaces for dynamic roll maneuvers, provided sufficient longitudinal control power remains.

As the considered UCAV configuration without vertical fins is expected to be unstable in the yaw axis, the dynamics in this axis are particularly considered in the flight dynamics assessment of this aircraft. In [29] requirements for the dynamics in the directional axis are suggested to assure that an unstable aircraft can be stabilized by an automatic flight control system. The author defines that the time to double the amplitude of the sideslip angle should be larger than $350 \mathrm{~ms}$. In this approach it is assumed that the dimensional side force derivatives with respect to sideslip angle and rudder deflection as well as the dimensional yaw moment derivative with respect to yaw rate in the aerodynamic coordinate system are negligible:

$$
Y_{\text {rud }} \approx N_{r_{a}} \approx 0 \text {. }
$$

This allows the following approximation for the time to double of the sideslip angle:

$$
T_{2}=\frac{\operatorname{acosh}(2)}{\sqrt{-N_{\beta_{a}}}},
$$

with $N_{\beta_{a}}$ representing the dimensional yaw moment derivative with respect to sideslip angle in aerodynamic axes. All dimensional derivatives are defined according to [30], i.e. correspond to the respective force or moment derivative divided by the mass or moment of inertia. Directional stability requires a positive value of the parameter $N_{\beta_{a}}$, given in the aerodynamic coordinate system. The dimensional derivative $N_{\beta}$ in body-fixed coordinates is negative for both aerodynamic datasets. However, the transformation into the aerodynamic coordinate system leads to a positive (i.e. stable) dimensional yaw moment derivative at the considered trim point because of the influence of the stable (i.e. negative) dimensional roll moment derivative with respect to sideslip angle $L_{\beta}$ :

$$
N_{\beta_{a}}=N_{\beta_{b}} \cos (\alpha)-L_{\beta_{b}} \sin (\alpha) .
$$

At the considered trim point with a Mach number of 0.3 the angles of attack of $6.3^{\circ}$ in case of the VSAERO based model and $7.4^{\circ}$ in case of the wind tunnel data based model are large enough to ensure a stable directional motion thanks to the influence of $L_{\beta}$. At larger Mach numbers the influence of $L_{\beta}$ becomes smaller due to the smaller angle of attack. Larger Mach numbers, however, cannot be reliably assessed with the available dataset because the wind tunnel data is only valid for the low speed range. An extrapolation of the available dataset to a Mach number of 0.5 with trimmed angles of attack of $3.3^{\circ}$ (wind tunnel dataset) and $2.7^{\circ}$ (VSAERO dataset) suggests that the UCAV would be slightly unstable at this Mach number with times to double amplitude of the sideslip angle of $3.4 \mathrm{~s}$ for the VSAERO based model and $3.0 \mathrm{~s}$ for the wind tunnel based model. Both values are well above value of the minimum time-to-double of $350 \mathrm{~ms}$ 
required in [29]. This suggests that the instability of the UCAV might still be acceptable at the Mach number of 0.5 . These results as well as the instability for the high speed range need, however, to be verified with appropriate aerodynamic data, which are valid for the corresponding Mach numbers.

What extent of instability can actually be compensated by an active flight control system depends on several parameters like available aerodynamic control power, moments of inertia, time delays, and actuator dynamics as well as the control system itself. The sensitivities with respect to these parameters will be analyzed in future studies.

\subsection{Discussion}

The results of the system identification process show that the new approach of combining innovative wind tunnel maneuvers with parameter estimation is an effective and efficient method to determine an aerodynamic model of an unconventional flying wing configuration. The analysis demonstrates a good match of the determined aerodynamic model with the wind tunnel measurements. The method was able to model the strongly nonlinear aerodynamics with its special characteristics dominated by vortex structures and is therefore evaluated as a valuable new approach in this context.

The main benefits compared to classical linear evaluation methods are the ability to model highly nonlinear aerodynamic behavior and to substantially reduce the number of wind tunnel experiments. Thus a complete 6 -DoF envelope can be generated within the wind tunnel hardware limits. The wind tunnel time saving depends on the test program, but can exceed $75 \%$. A processing of the new wind tunnel maneuvers is thereby only possible thanks to the combination with the nonlinear system identification. A further advantage of the new approach is that the resulting aerodynamic model can directly be used in flight dynamics simulation.

A still remaining disadvantage of the new approach is that it is - due to the currently available hardware - limited to low Mach numbers. The MPM at DNW-NWB restricts the Mach number range to low values of about 0.15 for the maneuvers presented in this paper because the loads become too strong for the internal balance otherwise. With improved hardware, however, the presented approach could also be extended to larger Mach numbers. A further inherent drawback of the wind tunnel investigations are the high costs resulting from a large number of experiments, e.g. to evaluate the effectiveness of various control surfaces. This makes it difficult to produce measurements over the whole flight envelope, which is necessary for comprehensive flight dynamics analysis of the aircraft

Analyses with simple and fast aerodynamic tools like VSAERO are much cheaper and thus allow a computation of the entire flight envelope as well as an easy comparison of different configurations at an early stage in the aircraft design process. However, especially for unconventional aircraft configurations like the present highly swept flying wing, the ability to correctly predict the aerodynamic behavior is very limited as the complex flow conditions including vortex structures and breakdown cannot be modeled.
The comparison of the two flight dynamics models with aerodynamics models derived from the dynamic wind tunnel experiments or determined with VSAERO shows that the application of linear flying qualities criteria yields some significant differences between the two aerodynamic datasets. In the longitudinal axis the VSAERO based model is much more agile than the wind tunnel data based model. This behavior results from the fact that positions of the aerodynamic neutral point differ for the two aerodynamic datasets. Concerning the lateral-directional dynamics of the UCAV, it could be noticed that the aerodynamic dataset determined with VSAERO shows better flying qualities for all criteria applied in the present analysis. Even if the differences for the lateral-directional eigenmodes are not very large, a more significant deviation between the dynamic behaviors of the two models could be revealed concerning the achievable roll performance. The VSAERO based model provides sufficient roll performance to achieve level 1 flying qualities as a highly maneuverable aircraft of class IV, whereas the wind tunnel experiment based aircraft model only reaches level 3 flying qualities. The observed effect that VSAERO overestimates the efficiency of the control surfaces was expected due to the underlying assumptions of inviscid and incompressible potential flow theory and the simplified linear superposition of separate control surface deflections specified in the employed CPACS interface. Nevertheless, the overestimation of the efficiencies always has to be kept in mind for the evaluation of the VSAREO based data

In order to improve the roll performance and achieve level 1 flying qualities for class IV aircraft as well, it should be considered to apply both trailing edge control surfaces on each wing for roll control - as far as permitted by persevering sufficient longitudinal control power - to increase the roll maneuverability of the UCAV. With the increase control surface size of the DLR-F19 model with $25 \%$ control surface depth an improved roll performance can be expected as well.

Altogether the flying qualities of the DLR-F19 configuration can be rated as satisfactory considering the fact that the configuration was analyzed without any kind of automatic flight control. A detailed analysis of the closed-loop system with active flight control still has to be performed for a final assessment of the configuration, however. This analysis is part of the ongoing studies at DLR.

The observed differences between the dynamics resulting from the two aerodynamic datasets support the assumption that low-fidelity aerodynamic computations with VSAERO are not sufficient to adequately model the aerodynamic behavior of such a flying wing configuration with highly swept leading and trailing edges and low aspect ratio. On the other hand, the results show that some critical issues of the configuration can already be identified with such simple methods, even if the absolute values show significant deviations from high-fidelity data. However, it is essential to be aware of the fact that the results obtained with VSAERO do not cover all relevant physical effects and might thus yield flying qualities that do not flawlessly match the aircraft dynamics in reality. 


\section{CONCLUSIONS}

The current paper presents an innovative way to derive a flight dynamics model from wind tunnel data by means of a system identification approach. This is based on the combination of the dynamic capabilities of the Model Positioning Mechanism (MPM) at DNW-NWB and the system identification expertise at the DLR Institute of Flight Systems. New quasi-steady maneuvers with superimposed harmonic excitation or frequency sweeps are applied via the MPM and then evaluated with a nonlinear parameter estimation tool. Thanks to these new maneuvers the wind tunnel time could be reduced up to $75 \%$. The finally elaborated aerodynamic model resulting from this approach showed a good match with the provided wind tunnel data and could directly be used for the flight dynamics model.

The comparison of the flying qualities of two flight dynamics models containing either the aerodynamic model of the system identification process or the aerodynamic parameters computed with VSAERO revealed significant differences between the two aerodynamic approaches. Even though this was expected because VSAERO is not designed for complex flow structures like the ones occurring at the considered DLRF19 highly swept flying wing, the simple aerodynamic approach could still indicate some major trends of the flight dynamic behavior of the configuration.

Nevertheless, costly high-fidelity CFD computations or wind tunnel experiments are required to extract accurate aerodynamics and flight dynamics for the appropriate design of a highly swept flying wing like the DLR-F19. A way to improve the efficiency of the process is the approach presented in this paper with innovative wind tunnel maneuvers followed by nonlinear system identification.

\section{Conflict of Interest}

The authors declare that they have no conflict of interest.

\section{REFERENCES}

[1] Cummings, R. M., Schütte, A.: An Integrated Computational/Experimental Approach to UCAV Stability \& Control Estimation: Overview of NATO RTO AVT-161, $28^{\text {th }}$ AIAA Applied Aerodynamics Conference, AIAA-2010-4392, Chicago, IL, USA (2010)

[2] Liersch, C. M., Huber, K.: Conceptual Design and Aerodynamic Analyses of a Generic UCAV Configuration, $32^{\text {nd }}$ AIAA Applied Aerodynamics Conference, AIAA-2014-2001, Atlanta, GA, USA (2014)

[3] Rohlf, D., Schmidt, S., Irving, J.: Stability and Control Analysis for an Unmanned Aircraft Configuration Using System-Identification Techniques, J Aircraft, Vol. 49, No. 6 (2012)

[4] Nathman, J. K.: VSAERO - A Computer Program for Calculating The Nonlinear Aerodynamic
Characteristics Of Arbitrary Configurations, Users' Manual Version 7.2, Redmond, WA, USA (2007)

[5] Nagel, B., Böhnke, D. Gollnick, V. , Schmollgruber P., Rizzi, A., La Rocca, G., Alonso, J. J.: Communication in Aircraft Design: Can we establish a Common Language?, $28^{\text {th }}$ International Congress of the Aeronautical Sciences, Brisbane, Australia (2012)

[6] Bergmann, A.: The New Aeroacoustic Low Speed Facility DNW-NWB, $18^{\text {th }}$ AIAA/CEAS Aeroacoustics Conference, AIAA-2012-2173, Colorado Springs, Colorado, USA (2012)

[7] Pott-Pollenske, M., von Heesen, W. , Bergmann, A.: Acoustical Preexamination Work and Characterization of the Low Noise Wind Tunnel DNW-NWB, $18^{\text {th }}$ AIAA/CEAS Aeroacoustics Conference, AIAA-2012-2175, Colorado Springs, Colorado, USA (2012)

[8] Loeser, T., Schröder, E.: The Anechoic Plenum of the DNW-NWB Aeroacoustic Wind Tunnel, $18^{\text {th }}$ AIAA/CEAS Aeroacoustics Conference, AIAA2012-2179, Colorado Springs, Colorado, USA (2012)

[9] Bergmann, A.: Modern Wind Tunnel Techniques for Unsteady Testing - Development of Dynamic Test Rigs, in: Hermann Schlichting - 100 Years, pp. 59-77, Springer, Braunschweig, Germany (2009)

[10] Cummings, R. M., Schütte, A.: The NATO STO AVT-201 Task Group "Extended Assessment of Stability and Control Prediction Methods for NATO Air Vehicles", $32^{\text {nd }}$ AIAA Applied Aerodynamics Conference, AIAA-2014-2394, Atlanta, GA, USA (2014)

[11] Vicroy, D., Huber, K., Rohlf, D., Loeser, T.: Lowspeed Dynamic Wind Tunnel Test Analysis of a Generic $53^{\circ}$ Swept UCAV Configuration, $32^{\text {nd }}$ AIAA Applied Aerodynamics Conference, AIAA-20142003, Atlanta, GA, USA (2014)

[12] Loeser, T.: Experimental Research of Dynamic Derivatives of Unsteady Moved Aircraft, in: Experimental Determination of Dynamic Stability Parameters, VKI LS 2008-02, Rhode-St-Genèse, Belgium (2008)

[13] Loeser, T., Rohlf, D.: Experimental Determination of Dynamic Derivatives in a Wind Tunnel using Parameter Identification, in: New Results in Numerical and Experimental Fluid Mechanics $X$, $19^{\text {th }}$ STAB/DGLR Symposium Munich, Germany (2014)

[14] Seher-Weiß, S.: User's Guide: FITLAB Parameter Estimation Using MATLAB - Version 2.0, DLRInternal Report, DLR-Internal Report, 111-2007/27 (2007)

[15] Fischenberg, D.: Identification of an Unsteady Aerodynamic Stall Model from Flight Test Data, 20th Atmospheric Flight Mechanics Conference, AIAA Paper 95-3438, Baltimore, MD, USA (1995)

[16] Jategaonkar, R. V.: Flight Vehicle System Identification: A Time Domain Methodology, Vol. 
216, AIAA Progress in Astronautics and Aeronautics Series, AIAA, Reston, VA, USA (2006)

[17] Kier, T., Looye, G., Scharpenberg, M., Reijerkerk, $M$.: Process, methods and tools for flexible aircraft flight dynamics model integration, International Forum on Aeroelasticity and Structural Dynamics, Stockholm, Sweden (2007)

[18] Looye, G.: The New DLR Flight Dynamics Library, in: Proceedings, $6^{\text {th }}$ Modelica Conference, 200803-03 - 2008-03-04, Bielefeld, Germany ( 2008)

[19] Kuchar, R., Steinhauser, R., Looye, G.: Design of Flight Control Laws for Agile and Highly Swept Aircraft Configurations, Deutscher Luft- und Raumfahrtkongress 2015, Rostock, Germany (2015)

[20] Nauroz, M., Becker, R., Robinson, J.: Individual Effects of Components on Cycle/Propulsive Efficiency for Variable Cycle Engines, Deutscher Luft- und Raumfahrtkongress 2012, Berlin, Germany (2012)

[21] Duus, G., Duda, H.: HQML: Ein Programmpaket zur Flugeigenschaftsanalyse unter MATLAB, DLRInternal Report, 111-97/22 (1997)

[22] Duus, G.: Überarbeitung des HAREM Programmpakets, DLR-Internal Report, 111-2001/10 ( 2001)

[23] Ehlers, J.: Flying Qualities Analysis of CPACS Based Aircraft Models, DLR-Internal Report, 1112013/21 (2013)

[24] N.N.: Mil-STD Military Standard, Flying Qualities of Piloted Aircraft, MIL-STD-1797A (1990)

[25] Schuette, A., Hummel, D., Hitzel, S. M. "Numerical and experimental analyses of the vortical flow around the SACCON configuration", 28th AIAA Applied Aerodynamics Conference, Chicago, Illinois, USA (2010)

[26] Liersch, C.M., Huber, K.C., Schütte, A., Zimper, D. Siggel, M.: Multidisciplinary Design and Aerodynamic Assessment of an Agile and Highly Swept Aircraft Configuration, Deutscher Luft- und Raumfahrtkongress, Rostock, Germany (2015)

[27] Tobie, H.N.: A new longitudinal handling qualities criterion, Boeing Company (1967)

[28] Huber, K., Vicroy, D., Schütte, A., Hübner, A.-R.: UCAV model design and static experimental investigations to estimate control device effectiveness and S\&C capabilities, $32^{\text {nd }}$ AIAA Applied Aerodynamics Conference, AIAA-20142002, Atlanta, GA, USA (2014)

[29] Osterhuber, R.: FCS Requirements for Future LO Combat Aircraft, STO/AVT-215 Workshop on "Innovative Control Effectors for Military Vehicles", Stockholm, Sweden (2013)

[30] Stevens, B., Lewis, F.: Aircraft Control and Simulation, John Wiley \& Sons, New York, USA (1992) 